\title{
Dynamics of large-scale electrophysiological networks: a technical review
}

George C. O'Neill't, Prejaas Tewarie ${ }^{1 \dagger}$, Diego Vidaurre ${ }^{2}$, Lucrezia Liuzzi', Mark W. Woolrich ${ }^{2}$, Matthew J. Brookes ${ }^{*}$

1 Sir Peter Mansfield Imaging Centre, School of Physics and Astronomy, University of Nottingham, Nottingham, United Kingdom

2 Oxford Centre for Human Brain Activity, Department of Psychiatry, University of Oxford, United Kingdom

10

Review for a special issue of Neurolmage

Word Count: $\quad 13,613$ (body) 5728 (references)

Citations: 224

Page count: $\quad 36$

20 Figures: 6

Tables: $\quad 1$

Boxes: 2

${ }^{\dagger}$ GCO and PT contributed equally

${ }^{*}$ Corresponding author:

40 Matthew J. Brookes

Sir Peter Mansfield Imaging Centre

University Park

Nottingham

NG7 2RD

Email: matthew.brookes@nottingham.ac.uk

Tel: $+44(0) 1159515188$ 
Abstract

For several years it has been argued that neural synchronisation is crucial for cognition. The idea that synchronised temporal patterns between different neural groups carries information above and beyond the isolated activity of these groups has inspired a shift in focus in the field of functional neuroimaging. Specifically, investigation into the activation elicited within certain regions by some stimulus or task has, in part, given way to analysis of patterns of co-activation or functional connectivity between distal regions. Recently, the functional connectivity community has been looking beyond the assumptions of stationarity that earlier work was based on, and has introduced methods to incorporate temporal dynamics into the analysis of connectivity. In particular, non-invasive electrophysiological data (magnetoencephalography / electroencephalography (MEG/EEG)), which provides direct measurement of whole-brain activity and rich temporal information, offers an exceptional window into such (potentially fast) brain dynamics. In this review, we discuss challenges, solutions, and a collection

60 of analysis tools that have been developed in recent years to facilitate the investigation of dynamic functional connectivity using these imaging modalities. Further, we discuss the applications of these approaches in the study of cognition and neuropsychiatric disorders. Finally, we review some existing developments that, by using realistic computational models, pursue a deeper understanding of the underlying causes of non-stationary connectivity.

Keywords: dynamic functional connectivity, magnetoencephalography, dynamic functional networks, electroencephalography, MEG, EEG. 


\section{Introduction}

The field of functional neuroimaging has undergone a paradigm shift over the past quarter century, evolving away from mapping tasked evoked areas of activation and towards identifying and characterising, either at rest or during a task, patterns of co-activation among spatially separate brain regions. More specifically, two or more regions are considered functionally connected if their activity profiles exhibit some statistical interdependency. Functional connections can be task-induced, where their cognitive or behavioral roles can be explicitly attributed, or in the absence of task (Engel et al., 2013), where their apparent function is more ambiguous. Since a breakthrough study by Biswal and colleagues (1995), where the blood oxygenation level dependent (BOLD) response of the bilateral primary motor cortices was found to be highly correlated, a robust set of large scale functional networks have been identified (e.g. Beckmann et al., 2005; Brookes et al., 2011b; Cole et al., 2014; de Pasquale et al., 2012; Fox et al., 2005; Luckhoo et al., 2012; Raichle et al., 2001; Shulman et al., 1997; Smith et al., 2009; Stevens, 2016). These networks are of significant clinical interest in view of the mounting evidence that they are compromised in neurological disease (e.g. Friston, 1998; Kessler et al., 2014; Nugent et al., 2015; Palaniyappan and Liddle, 2012; Schnitzler and Gross, 2005). As the field of functional connectivity continues to mature, and we better characterise these networks, we introduce an opportunity to discover why network connections are perturbed in pathology. Exploiting these findings gives us the opportunity to move connectivity analysis from a tool for neuroscientists, into a biomarker for diagnosis, providing an objective measure of illness severity (Brookes et al., 2016), or even tracking the response to clinical intervention (Carbo et al., 2017). However to achieve this goal, further work is required to generate a true understanding of the interaction between network connections and the behaviour that they support.

A large body of previous work has successfully characterised the spatial features of neural connectivity (Bastos and Schoffelen, 2015; Fox and Raichle, 2007; Friston, 2011; O'Neill et al., 2015a; Schoffelen and Gross, 2009), however many of these studies have ignored the temporal dimension of the data, i.e. when and how the strength of connectivity between regions varies across the experiment. It is generally accepted that modulations in communication between neuronal populations lie at the very

100 heart of human cognition (Fries, 2015), with communication assumed to be mediated by neuronal oscillations (i.e. communication through coherence). Since neural oscillations modulate very quickly (Pfurtscheller and Aranibar, 1977; Pfurtscheller and Lopes da Silva, 1999), it therefore follows that connectivity should also change rapidly, especially in response to sensory and cognitive cues. An adequate description of dynamic connectivity will thus be crucial if we are to elucidate the nature of how cognitive processes are supported by networks. For example, time-locked oscillatory responses in typical experiments usually last on the order of several hundred milliseconds to a few seconds. The occurrence of these responses in relation to a stimulus is a crucial aspect to labelling these responses as, for example, top-down or bottom-up, or as feedforward or feedback processes. Static connectivity approaches have been applied widely to analyse the coordination of brain regions during a specific 110 cognitive demand (Hampson et al., 2006; Lamme, 2003; Liddle et al., 2016; Peled et al., 2001). However, in contrast to dynamic measures, these static approaches are not time-resolved and therefore cannot disentangle the pipeline of information processing in the brain. Therefore a dynamic approach to connectivity could bring fundamental insight into the spatial integration of feedback and feedforward processes in the brain.

Most research on functional connectivity has been performed using functional Magnetic Resonance Imaging (fMRI) due to the maturity and relatively widespread availability of this technology. However, the BOLD response is only a proxy for brain activity, and the sluggish nature of this haemodynamic signal can obfuscate rapid neuronal dynamics. fMRI has been used to study the dynamics of functional

120 connectivity (Hutchison et al., 2013; Preti et al., 2016), but the time windows within which connectivity can be calculated with a reasonable degree of certainty are typically a minimum of thirty seconds, and are therefore not adequately time-resolved to address the question of network dynamics (Allen et al., 2014). However, thanks to their excellent temporal resolution and good spatial coverage, 
electrophysiological modalities open up an opportunity to measure neuronal oscillations and assess directly the fast fluctuations in neuronal coherence which lies at the very heart of dynamic communication in the brain. More specifically, electrocorticography (ECoG; Penfield and Jasper, 1954), magnetoencephalography (MEG; Cohen, 1972) and electroencephalography (EEG; Berger, 1929) are in a unique position to interrogate the shortest temporal scales of functional connectivity, with the encephalographic measures in particular being able to cover much of the cerebral cortex. Invasive electrophysiological recordings have managed to capture time-dependent correlations between oscillations in the mouse brain since before the turn of the millennium (MacDonald et al., 1996). However, it is only relatively recently that we have been able to reliably do this non-invasively with whole-cortex coverage in humans (de Pasquale et al., 2010; Liu et al., 2010). In summary, by investigating the dynamics of connectivity in the brain at the time fast scales that are accessible in electrophysiological data and commensurate with cognition, we have a unique opportunity to access how these connections evolve in time and how they may be perturbed by disease.

In this review, we look at the current state of dynamical electrophysiological connectivity research in both sensory/cognitive tasks and the resting state, with a particular emphasis on non-invasive $140 \mathrm{MEG} / \mathrm{EEG}$ measures, although these methods should also be applicable (or easily adaptable) to invasive data. The paper is organised as follows: Section 2 is a summary of some of the methodology required to measure functional connectivity in the static domain. Section 3 introduces a simple and popular method for capturing connectivity dynamics, the sliding window. We cover methodological considerations, as well as neuroscientific and clinical applications of this framework. However, the sliding window has significant limitations and so in Section 4 we look at current alternative methods to overcome such issues, along with examples of experimental findings. Finally, simply measuring dynamic connections, whilst powerful, cannot capture the biophysical processes underlying connectivity, so we need to use these observations in conjunction with computational models to explain our findings. Section 5 therefore looks at the current state of the modelling literature with respect to generating connectivity dynamics and explains how these findings are beginning to inform us about the underlying mechanisms of neural communication. 


\section{Measurement of static connectivity: a recap}

\subsection{Connectivity Metrics}

A functional connection in the brain is defined typically as the temporal correlation between remote neurophysiological events' (Friston, 1994). However, the rich temporal structure of electrophysiological data warrants an expansion of this definition to include other types of 'statistical interdependencies' between two or more sources. The number of approaches to assess static connectivity has been steadily growing, with many studies assessing their strengths and weaknesses (Brookes et al., 2011a; Colclough et al., 2016; Garcés et al., 2016; Hassan et al., 2017; Liuzzi et al., 2016; Pereda et al., 2005; Wang et al., 2014). As choosing the correct metric from such a wealth of options may be cumbersome, we here propose a few simple criteria to guide the selection of the best-suited metric for a given data and research question. First, do we intend to assess relations between the phase, or the amplitude of neural activity? Second, are we assuming a linear relation between the time courses or an approach which can also account for non-linearity? Third, are we interested in directionality of information flow between the sources? Table 1 lists some popular metrics to assess connectivity, and specifies their place in relation to these criteria. Other measures such as phase-amplitude coupling (Canolty et al., 2006; Florin and Baillet, 2015; Samiee and Baillet, 2017) and generalised synchronisation (Stam, 2004) are also interesting for the analysis of dynamic connectivity but they do not necessarily fit this simple categorisation. Particularly in MEG/EEG investigations, we also strongly recommend that functional

170 connectivity analysis is performed at the source, rather than sensor level, as this removes many of the ambiguities associated with sensor level analysis (Schoffelen and Gross, 2009). In addition, sensitivity of functional connectivity detection may be improved by reconstructing multiple sources at the same location in multiple orthogonal orientations (i.e. a vector reconstruction; Huang et al. 2004). This is because typically selecting a single source orientation is usually based on the orientation of maximal signal variance (Johnson et al., 2011) and this is not necessarily the orientation of maximum connectivity. Some studies have adopted this multi-orientation approach and demonstrated improved measurements of connectivity (e.g. Marzetti et al., 2013).

\subsection{Controlling for signal leakage}

180 A problem of source reconstruction in MEG/EEG is that the sources may not be fully spatially resolved, but rather are smeared out across a relatively large brain volume. This problem arises primarily from inaccurate forward models and the ill-posed nature of the inverse MEG/EEG problem, which projects data from relatively few sensors to many possible source locations. This can result in two uncorrelated sources having their reconstructed time courses erroneously correlated. If ignored, this can artificially inflate the level of connectivity between two sources. The way leakage propagates across the source space is non-trivial, and solutions need to be implemented to reduce this effect on functional connectivity. Multiple solutions exist, though all are currently based on the same principle: leakage propagates instantaneously due to inverse reconstruction being time independent, so any zero-lagged relationship between two sources must be discounted or removed from the data entirely. Obviously,

190 this does come at the expense of removing genuine zero-lag connectivity, which has been shown to be relevant in the invasive literature (Singer, 1999).

The simplest method to reduce the influence of leakage on the estimation of connectivity is to use a leakage-invariant metric. For example, Imaginary Coherence (Nolte et al., 2004) uses only the imaginary part of the cross spectral density, ignoring zero and $\pi$-phase relationships and thereby removing zero-lag effects. The Phase Lag Index (PLI; Stam et al. 2007), builds upon the popular Phase Locking Value metric (Lachaux et al., 1999) by looking at the asymmetry of the distribution of phase differences, which can only be explained by non-zero phase lags. Other metrics use more involved processing to effectively minimise the effect of leakage. For example, Transfer Entropy (Schreiber,

2002000 ) uses conditional probabilities to regress out zero-lag relations. A few other metrics with automatic control for leakage are also listed in Table 1. 
Nevertheless, many common metrics are sensitive to leakage and require a correction prior to their application. The simplest method to reduce leakage is to orthogonalise the data by performing a pairwise linear regression between a seed and test signal (Brookes et al., 2012b; Hipp et al., 2012). The orthogonalisation method can be extended into the multivariate domain to correct for leakage between two clusters of voxel timecourses, or multidimensional source timecourses generated from vector source reconstruction (Brookes et al, 2014, O'Neill et al 2015). For all-to-all connectivity analysis (where connectivity is assessed in a pairwise manner between each and every region pair), it is possible to use a systematic approach of sequential pairwise regressions between all nodes such that all signals are made orthogonal (Brookes et al., 2016; Hunt et al., 2016; Maldjian et al., 2014). However, such methods still result in so-called secondary leakage or ghost connections (Palva and Palva, 2012), where a genuine connection between two nodes spreads in space due to leakage from these nodes to other proximal regions. An elegant solution to this problem was proposed by (Colclough et al., 2015) where the entire set of regions of interest are orthogonalised symmetrically in one single computation using Löwdin Orthogonalisation (Löwdin, 1950). However, it is important to note the maximum number of timecourses which can be orthgonalised this way is limited to the rank of the data. Typically, this would correspond to the number of recordings (sensors or electrodes) from an experiment, but software processing such as Signal Space Separation (Taulu et al., 2004) can reduce this figure further.

It is important to note that these leakage reduction methods do not ameliorate all effects on functional connectivity entirely. The limitations of these methods, in particular orthogonalisation, come from some of the technical assumptions required in the implementation. First, they require the raw data prior to correction to be Gaussian distributed (Brookes et al., 2014), which is not necessarily true. Secondly they also assume a constant signal to noise ratio (SNR) across time and frequency. The effects of varying SNR across frequency has not (to our knowledge) been investigated systematically. However in practice, the effectiveness of leakage reduction can be maximised by performing leakage reduction after bandpass filtering the data into the band of interest.

230 Finally, it is possible to correct for leakage prior to the generation of source time courses by modifying the forward and inverse solutions. There are methods to calculate cross-talk functions (CTFs; (Farahibozorg et al., 2017; Hauk and Stenroos, 2014; Hauk et al., 2011) between sources and modify the inverse solution accordingly (DeFLeCT; Hauk and Stenroos 2014). Wens and colleagues (2015) take this idea one step further by correcting the forward solutions, ensuring that zero-lagged relationships of reconstructed sources in the immediate vicinity of the test region of interest are removed entirely. (Wens et al., 2015). In summary, there are a number of different methods for leakage reduction. These methods have been shown to be critical when computing static connectivity and they prove equally critical in estimating dynamic connections. 


\begin{tabular}{|c|c|c|c|c|c|}
\hline Connectivity Metric & Type & Relation & Directed? & Assumes Linearity? & Leakage Control? \\
\hline Amplitude Envelope Correlation (Liu et al., 2010) & Amplitude Coupling & Marginal or Partial & No & Yes & No \\
\hline $\begin{array}{l}\text { Leakage controlled (orthogonalised) Amplitude Envelope } \\
\text { Correlation } \\
\text { (Brookes et al., 2012b; Colclough et al., 2015; Hipp et al., } \\
\text { 2012) }\end{array}$ & Amplitude Coupling & Marginal or Partial & No & Yes & Yes \\
\hline Coherence (Nunez et al., 1997) & Spectral Coherence & Marginal or Partial & No & Yes & No \\
\hline Imaginary Coherency (Nolte et al., 2004) & Spectral Coherence & Marginal or Partial & No & Yes & Yes \\
\hline Phase Locking Value (Lachaux et al., 1999) & Phase Coupling & Marginal & No & No & No \\
\hline Phase Lag Index (Stam et al., 2007) & Phase Coupling & Marginal & No & No & Yes \\
\hline Weighted Phase Lag Index (Vinck et al., 2011) & Phase Coupling & Marginal & No & Yes & Yes \\
\hline Phase Slope Index (Nolte et al., 2008) & Phase Coupling & Marginal & Yes & Yes & Yes \\
\hline Phase Difference Derivative (Breakspear et al., 2004) & Phase Coupling & Marginal & No & Yes & No \\
\hline Mutual Information (Paluš, 1997) & Amplitude or Phase Coupling & Marginal & No & No & No \\
\hline Transfer Entropy (Schreiber, 2000) & Amplitude or Phase Coupling & Marginal & Yes & No & Yes \\
\hline Granger Causality (Granger, 1969) & Autoregressive Modelling & Marginal & Yes & Yes & Disputed \\
\hline $\begin{array}{l}\text { Partially Directed Coherence (Baccala and Sameshima, } \\
\text { 2001) }\end{array}$ & Autoregressive Modelling & Partial & Yes & Yes & Disputed \\
\hline Synchronisation lilekihood (Stam and van Dijk, 2002) & Generalised synchronization & Marginal & No & No & No \\
\hline
\end{tabular}

Table 1: Some conventional connectivity metrics used on electrophysiological data which could be used in a dynamic and their associated properties. Tabled adapted with permission from Colclough et al (2016). 


\section{Measurement of dynamic connectivity}

There are numerous approaches to generating time evolving connectivity estimates from experimental data. However, they all have the same basic requirement: to infer how connected two ROIs are, multiple time points of data must be considered. How many time points are required is still an ongoing area of research which is discussed later, but in short there are two approaches to aggregating data to infer connectivity. The first, is to use multiple successive timepoints, whether that is using a temporal window (as in Section 3.1) or using other techniques (Section 4), this type of analysis is used extensively in experiments with no trial locking (such as resting state paradigms). Alternatively, if using multiple task trials, it is possible (in some cases) to infer connectivity across trials by aggregating across the same time point of multiple trials to generate connectivity dynamics. Whether that is by using a single sample from each trial (assuming it is the same point in time relative to a trial onset) or windows of data is dependent to the metric at hand.

\subsection{The sliding window approach}

The simplest way to assess dynamic connectivity is by using 'static' connectivity measures, similar to those in Table 1, within a sliding window framework. As illustrated in Figure 1, the time series of activity is segmented into time windows of width $d$, such that a window centered on time point $t$ has window boundaries at $t \pm 0.5 \mathrm{~d}$. The window is then moved forward in time a certain step $\mathrm{s}$, connectivity is assessed in this new window, and the process is repeated so that we can generate a time course of connectivity. The power of the sliding window is, that due to its fundamental similarities to a static analysis, most 'conventional' connectivity metrics in Table 1 should be compatible with it with little alteration, making it a flexible approach. However, one parameter selection which is not trivial, is the window length, with different connectivity metrics requiring differing lengths to adequately assess connectivity (e.g, it has been shown that phase lag metrics typically require more data to show consistent results than amplitude metrics; Colclough et al, 2016). That said, the sliding window is a popular and simple method that has been used in both the fMRI (Allen et al., 2014; Chang et al., 2013; Elton and Gao, 2015; Hutchison et al., 2013; Kucyi and Davis, 2014; Preti et al., 2016; Tagliazucchi et

270 al., 2012) and MEG/EEG literature (Baker et al., 2012; Betti et al., 2013; Brookes et al., 2011a; Brookes et al., 2014; Brovelli et al., 2017; Carbo et al., 2017; de Pasquale et al., 2010; de Pasquale et al., 2016; Doron et al., 2012; Lee et al., 2017; O'Neill et al., 2015b; O'Neill et al., 2017; Yang et al., 2012). 


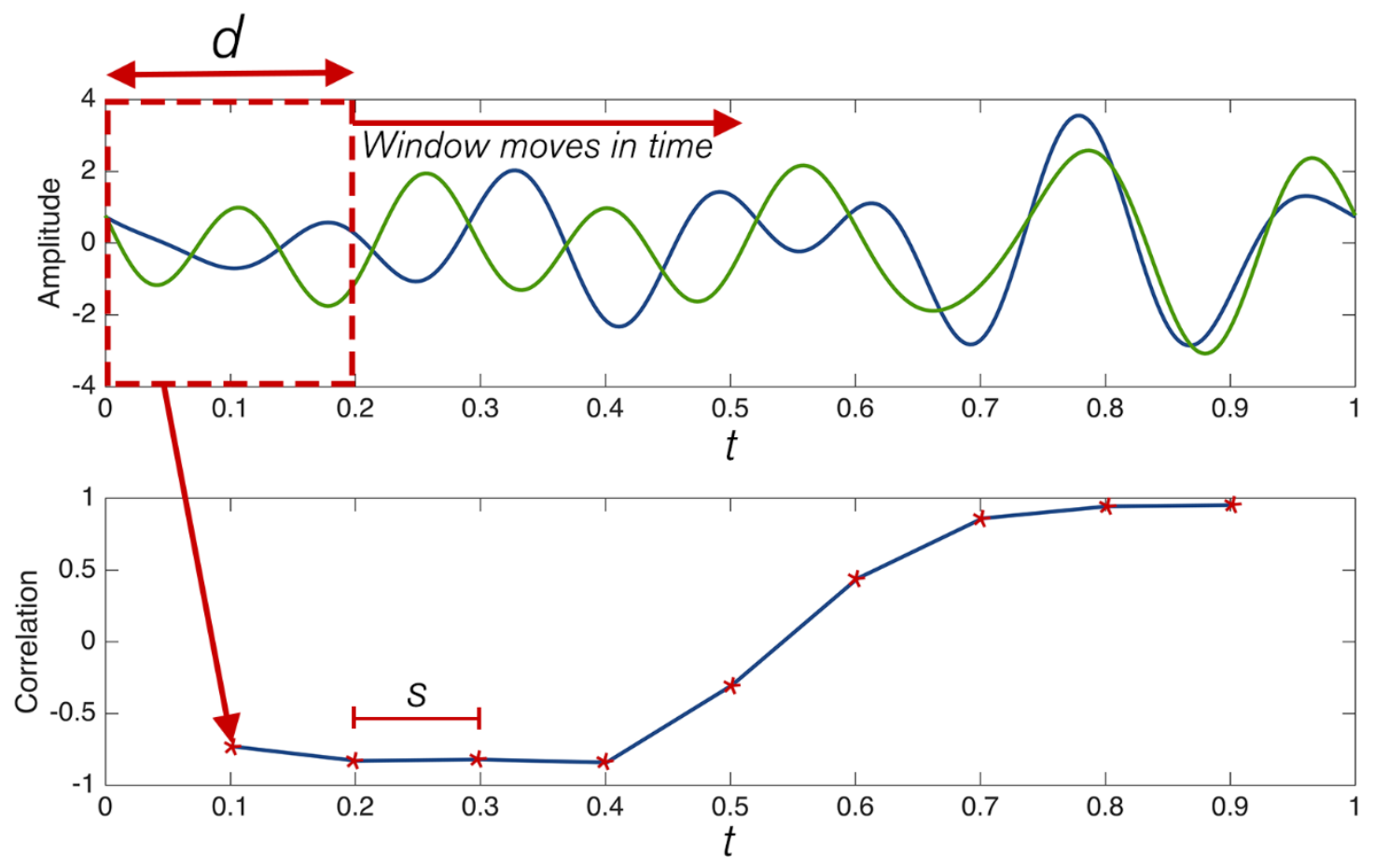

Figure 1: A cartoon describing the sliding window method to assess connectivity dynamics. Connectivity is assessed over a $d=0.2 \mathrm{~s}$ wide window (top panel, red dashed rectangle). Connectivity (in this case, correlation) can be assessed between the two windowed timecourses and the result is represented on the bottom panel as the asterisk at $t=0.1 \mathrm{~s}$. The window is allowed to move in steps of $s=0.1$ and the process is repeated to generate a timecourse of connectivity. Here this reveals a period of strong anticorrelation between the timeseries, followed by strong positive relation.

\subsection{Leakage correction revisited}

It is usual practice to control for leakage on the entire data set in a single step (see the static reduction flowchart in Figure 2A). However, as shown by O'Neill and colleagues (2015b) both analytically and in simulation, if the variance of the source time courses changes between windows, so does the effect of leakage. Figure 2B-C shows simulation results from O'Neill et al. (2015b); which show that in a null simulation where no zero-lagged relations between the seed region (blue dot) and the test regions (red volume) should exist, leakage is not entirely ameliorated if you do not control for leakage on the temporal scale at which connectivity is assessed on. In view of these results, we conclude that leakage needs to be reduced within every window in which connectivity is assessed rather than over the entire data set, as illustrated in the dynamic correction flowchart in Figure $2 \mathrm{~A}$.

\subsection{Identification of repeating patterns of connectivity/co-varying network patterns}

When studying dynamic connectivity, one typically assesses connectivity between all possible regions of interest and within many time windows. This means that thousands of connectivity matrices are typically estimated, calling for an automatic method to aid analysis of the results. Many methods have been proposed to find patterns in the set of connectivity matrices that are interpretable and functionally meaningful. Many of these methods are based upon the assumption that connectivity manifests itself in recurrent or repeating (spatial or temporal) patterns. 

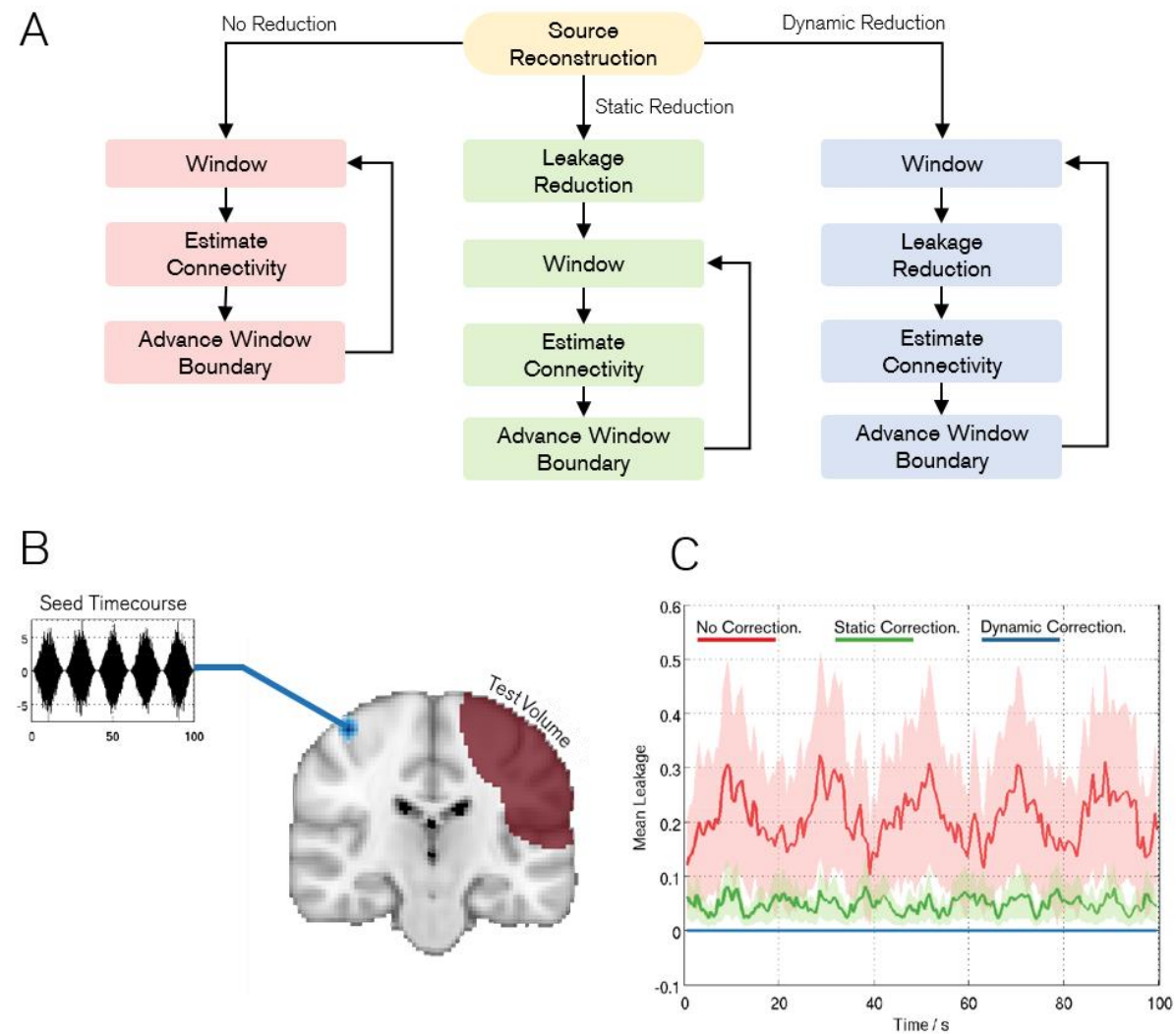

Figure 2: The dynamics of signal leakage. A) A flowchart illustrating the pipelines of sliding window connectivity analysis with no (red), static (green) and dynamic (blue) leakage reduction. B) A simulation in which a source is placed in the left hemisphere (blue dot) and the extent of the zero-lag relation between it and locations in the red test volume in the opposite hemisphere is measured. C) The results of the simulation, showing clear temporal structure to the leakage which is only completely eliminated when dynamic leakage reduction is applied. Panels $B$ and C are adapted with permission from O'Neill et al (2015).

K-means clustering (MacQueen, 1967) looks for groups or clusters of data points (typically adjacency matrices) that are proximal to each other in a multivariate space. In this case, a cluster will contain connectivity maps with similar topologies (or repeating spatial patterns of connectivity) across time. Kmeans allows for each "snapshot" of connectivity to be categorized into one of a (user-defined) number of 'states', where each state corresponds to a set of connection patterns. This at first may appear unintuitive to collapse down an entire experiments worth connectivity results into a handful of networks if we want to investigate their dynamics. However, each state is accompanied with a binary timecourse, and as seen in Figure 3A, if we were to average these timecourses across trials, we can generate a probabilistic timecourse, which describes the likelihood of any given network 'occurring' at a specific point in a trial (Figure 3B). This method has often been used in $\mathrm{FMRI}$ analyses since a study by Allen et al. (2014), and has also been shown to work in both MEG (O'Neill et al, 2015) and EEG (Mheich et al, 2015; Hassan et al, 2015). In a similar vein, Hutt and colleagues introduced a variant of k-means designed to work on cyclic data, such that clustering can be performed on phase information extracted from neuronal oscillations instead of using the connectivity profiles (Hutt et al., 2003). K-means approaches have some limitations however, the first being an a-priori knowledge of the number of states is required. Secondly k-means assumes that all of the connectivity profiles it is presented with (i.e. all windows) can be catagorised, which may not be the case and finally, it assumes mutual exclusivity of the states (i.e only one network is 'active' at a given point in time). 
A
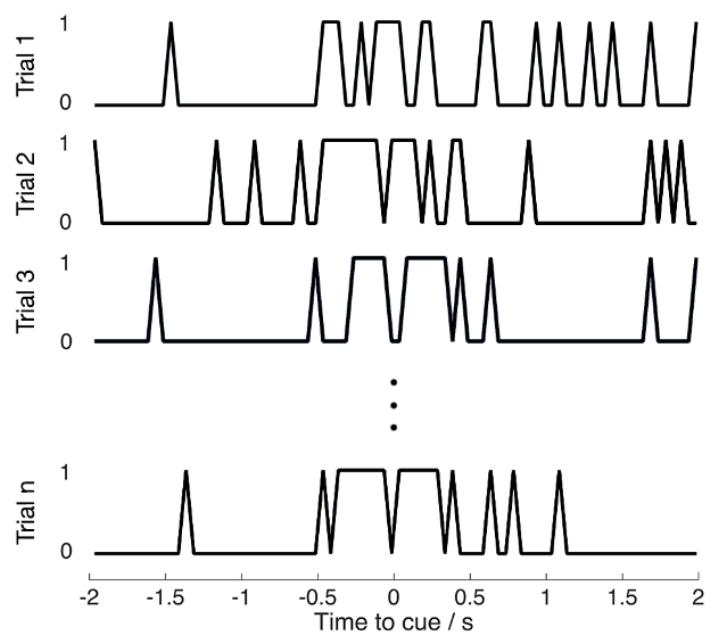

B

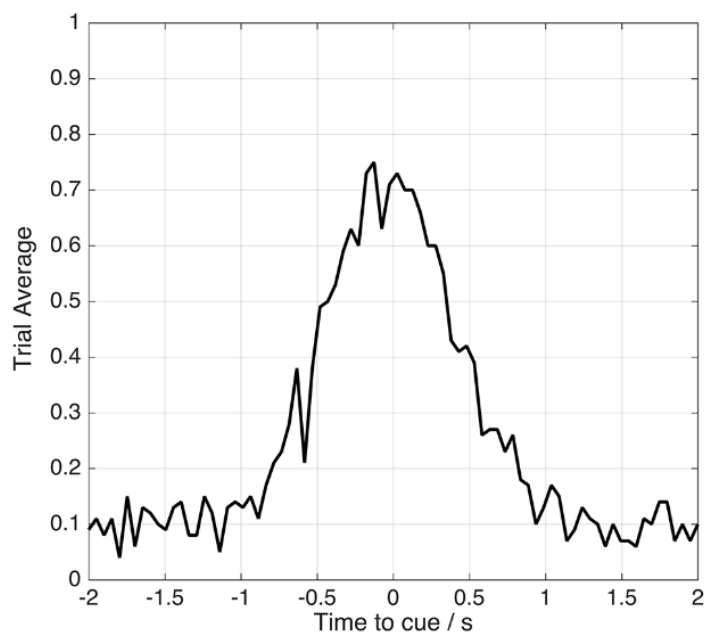

Figure 3: Binary timecourses in connectivity. A) Timecourses from (for example) k-means clustering which gives a binary representation of whether a state was 'active' or 'inactive'. These results themselves are not easily interpretable, but if each time series is averaged across trials (as in panel B) it can reveal dynamics in connectivity in the trial average. In this case it reveals a functional connection is most likely to occur when a cue is initiated.

Another popular approach, Independent Component Analysis, has been used extensively to find functional networks based on common activation time courses in MEG/EEG (Brookes et al., 2012a; Brookes et al., 2011b; Hall et al., 2013; Knyazev et al., 2016; Koelewijn et al., 2017; Koelewijn et al., 2015; Luckhoo et al., 2012; Nugent et al., 2015). In the case of dynamic connectivity, instead of applying independent component analysis to activation time courses, one could also apply it to a set of connectivity time courses (e.g. from sliding windows) to identify networks based on common modulations in connectivity between regions. The number of 'unique' connectivity timecourses in a dynamic connectome will likely be lower than the number of connections measured. ICA exploits this by extracting common connectivity timecourses across multiple connections between nodes. In this type of analysis, each independent component timecourse represents the temporal evolution of connectivity (O'Neill et al 2017) whilst the ICA mixing matrix describes a set of connections which modulate in a similar way and hence represents a network in which all connections share a similar behaviour. This allows for identification of networks which overlap spatially to be unmixed if each topography has a different temporal signature (O'Neill et al 2017).

Other methods based on signal decompositions include (but are not limited to) principle component analysis (Leonardi et al., 2013), dictionary learning (Grandjean et al., 2017), tensor decomposition methods (Escudero et al., 2015) and dynamic community detection (Bassett et al., 2013; Mucha et al., 2010). The latter two methods, tensor decomposition approaches and dynamic community detection, have recently been applied in the field of complex networks (Gauvin et al., 2014). Tensor decomposition approaches, such as non-negative tensor factorization, can be regarded as higher order variants of principal component analysis, where instead of vectors, algorithms are fed with tensors (e.g. concatenated time varying connectivity matrices). Likewise, this results in different subnetworks characterised by their activity time course. This approach has been applied to fMRI data and to models of phase oscillators, which allows us to extract similar time evolving subnetworks as ICA (Ponce-Alvarez et al., 2015). Community detection is the extension of the widely applied community detection algorithm in static networks (Newman, 2010), which has also been shown to reveal similar subnetworks as resting-state networks obtained from independent component analysis (Crossley et al., 2013; Meunier et al., 2009). The idea is to optimise a graph theoretic function, called modularity, which quantifies within 
community connections relative to connections between communities (Basset et al., 2013; Mucha et al 2010). This results in an assignment of every node in the network to a community. However, the open question remains whether all these different approaches to extract reoccurring patterns of connectivity capture similar or complementary spatiotemporal information. Are all approaches sensitive to network changes occurring at the same time-scale or do we need a set of pattern identification approaches to detect network modulations occurring at different timescales? These questions will need to be addressed in future research.

\subsection{Statistical inference}

Perhaps the greatest difficulty when assessing the dynamics of functional connectivity is determining quantitatively whether or not a change in connectivity between windows is due to a genuine nonstationary process, or simply a result of stochastic noise on the measurement. Indeed, it has been shown that a stationary connection can be artefactually deemed dynamic because of changes in the SNR of experimental data (Betzel et al., 2016; Hindriks et al., 2016; Lindquist et al., 2014). Therefore, adequate statistical testing needs to be applied to assess which changes in connectivity are truly meaningful. Statistical testing of dynamic data has extra computational complications over its static counterpart due to the number of time points we need to test.

In task data, the solution proposed by Maris and Oostenveld (2007) to test for significant changes in power in MEG/EEG can be adapted to accommodate dynamic connections. The idea is to perform significance testing on the trial-averaged connectivity time course, such that a time-evolving nonparametric null distribution can be generated using pseudo-trials. For example, generating pseudo-trial averages based on random trial onset times allows us to test whether the start of a trial or onset of a cue significantly deflects levels of connectivity and when significance occurs. This can also be applied to other null-hypotheses, such as testing for differences in connectivity between groups, where each permutation of the null distributions involves randomly assigning each trial or participant to one of two conditions. If connectivity time courses are extracted using ICA, a similar non-parametric method based on "sign flipping" can be implemented (Hunt et al., 2012; O'Neill et al., 2017; Winkler et al., 2014). The idea is to generate a surrogate time course by randomly selecting portions of an independent component (e.g. corresponding to half the participants), multiplying that section by -1 and then looking at the trial averaged component. By repeating this process multiple times, we can again generate a null distribution which evolves over time. The reasoning behind this idea is that, if there is a change in connectivity at a given point of the trial which is not present across all subjects, then the surrogate time courses would have the same magnitude as the real trial-average. Conversely, if the effect were genuine, the surrogate time course would have a significantly diminished magnitude (from timecourses cancelling out) and so the genuine timecourses would exceed the null threshold.

For situations where trial averaging or time locking cannot be implemented (such as in resting-state analysis), testing the confidence in a time varying connectivity estimation from (for example) sliding windows becomes more difficult. What you can test for is whether the fluctuations seen in a connectivity timecourse are spurious measurements or are a genuine observation. Here one would test against the null hypothesis, that connectivity across the entire experiment is static, and apparent connectivity

410 dynamics can be explained by an inability to precisely measure this when using a portion of the time series. To test this hypothesis, one has to construct surrogate data with the same covariance structure (i.e. maintaining the same level of static connectivity) and measure a given statistic of the surrogate connectivity time series, for example variance (such as in Hindriks et al., 2016), or perhaps the connectivity level in a given window. Should the respective test statistic of the original connectivity timecourse exceed the null, then the null hypothesis can be rejected. Whist there is no universal permutation method to generate surrogate data, there currently two popular ways which have been used in respect to connectivity dynamics. Phase randomization (Prichard and Theiler, 1994) of the original test data (Betzel et al., 2016; Brookes et al., 2014; Hindriks et al., 2016) is one such method. This has been shown to be effective for choosing a significance threshold in MEG data (Brookes et al 
420 2014), but phase randomization necessarily assumes Gaussianity in the original data, unless specific adjustments are made as in Betzel et al. (2016). Alternatively, one can model each time course of activity with a Vector Autoregressive Model (VAR) and, from there, generate a surrogate dataset which can have a coloured spectra (Chang and Glover, 2010; de Pasquale et al., 2016; Zalesky et al., 2014), however one has to ensure the model order is carefully chosen so tests do not return false positives or negatives. Crucially, what we are not testing for here is whether a connectivity timecourse is stationary or not in the strict statistical sense, but merely a confidence in the observations. As shown in a recent paper Liégeois and colleagues (2017), rejecting the null hypothesis in this way does not unequivocally prove that connectivity is non-stationary, but could also be due to nonlinear and/or non-Gaussian data. The field of testing for connectivity fluctuations in the resting state and interpreting the findings is not 430 trivial, but the aforementioned paper by Liégeois et al. (2017) serves as a helpful primer on these topics. This remains one of the most critical areas for future investigation.

\subsection{Findings using sliding window approaches}

Sliding window approaches for dynamic connectivity have been applied in basic neuroscientific and clinical studies. For example, de Pasquale et al (2010) used sliding windows to measure connectivity in the default mode network (DMN) at rest. First, they found the correlation between nodes would strongly fluctuate throughout the course of an experiment. There would be epochs where the entire DMN would be connected, followed by periods where only a subset of regions were connected. As a result, they found that network topography could be better resolved when selecting windows exhibiting

440 the strongest correlations during a recording rather than using a static window representing the entire experiment. In a follow up study, de Pasquale and colleagues (2012) investigated whether other resting state networks shared the same dynamic properties. They found that when the DMN nodes exhibited high intra-network connectivity, it would be seen to strongly connect to nodes of other networks typically seen in resting state paradigms (de Pasquale et al., 2012). This behaviour of the DMN cross-interacting with other networks supports the idea that that the DMN is a cortical hub which functional processing is routed through. Pushing this one step further, dynamic connectivity matrices from a sliding window analysis, when subject to a graph theoretical analysis, revealed three distinct hubs (default mode, dorsal attention and motor networks) which can dynamically allow between-network communication through them at rest (de Pasquale et al., 2016). A recent study went beyond quantification of hubs and quantified

450 the dynamics of the participation coefficient, local clustering and efficiency of temporal EEG networks using a sliding window approach based on PLV (Kabbara et al., 2017), this study also showed that the communication and integrations of these hubs could also be reliably detected using windows which were sub-second in width.

Sliding window approaches have been applied in some experiments aiming to understand primary sensory processes, i.e. in auditory, sensorimotor, and visuomotor studies (Baker et al., 2012; Betti et al., 2013, Brovelli et al., 2017; O'Neill et al., 2015b; Yang et al., 2012). In a recent visuomotor MEG study, participants were asked to perform a finger movement based on a visual cue (Brovelli et al., 2017). The authors used a sliding window approach and a dynamic community detection algorithm in

460 order to quantify three temporally-evolving subnetworks that showed peak activations at different time points in relation to stimulus onset. These were a visual-parietal subnetwork emerging before finger movement, followed by a subnetwork comprising sensorimotor and fronto-parietal regions, and by a large subnetwork with strong sensorimotor activation. In another visual MEG study (Yang et al., 2012), the authors analysed the neural processing of motion perception of an object moving towards a subject versus an object moving away from a subject. Using a sliding window approach in conjunction with mutual information, the authors demonstrated the presence of a different temporally resolved top-down connectivity for the two conditions. Another investigation into the dynamics of the visual cortex by Betti and colleagues (2013) found that not only was there a reduction amplitude envelope coupling in the visual network during movie watching, but transient reductions in connectivity were time locked to 470 psychophysical event boundaries in the movie (Betti et al., 2013). Studying the sensorimotor network, Brookes et al (2011a) showed significant fluctuations in the strength of functional connectivity between 
motor cortices in a sliding window analysis in the resting state. This result was extended by Baker et al (2012), who found that the motor network exhibits bi-stable dynamics, where periods of high connectivity were interspersed in between near-zero levels of connectivity. In a more recent study using K-means clustering (O'Neill et al., 2015b), the authors showed that could untangle the sensorimotor network into a set of smaller subnetworks that rapidly form and dissolve during a self-paced motor task. In particular a bilateral primary motor subnetwork was shown to have a high probability of 'activating' around the time of the button press, followed by a separate bilateral primary somatosensory network. This study illustrated that the sensorimotor network, while appearing as a single network in a static connectivity framework, is instead composed of different subnetworks with their own spatial and temporal dynamics.

The importance of transient functional networks has also been demonstrated during more demanding cognitive tasks, such as working memory and language processing tasks. A study by O'Neill et al (2017) showed large scale networks being recruited dynamically to individual stages of a Sternberg working memory paradigm. Large scale networks were derived from ICA on connectivity time courses. Networks identified included the visual network during presentation of the stimuli, a language network incorporating Wernicke's and Broca's areas during the recall phase, multiple parietal to occipital connections which occurred during encoding and recall phases, and somatosensory networks when a 490 button was pressed confirming matching stimuli. In a separate study, using sensor level MEG data, lexicon stimuli were presented to the participants and dynamic network configurations were analysed in relation to the "word" vs "pseudoword" conditions (Doron et al., 2012). The authors used a sliding window approach using the amplitude envelope correlation as connectivity metric in combination with a dynamic community detection method in order to separate subnetworks in a time-resolved fashion. They showed that modulations in subnetworks were associated with changes in inter-hemispheric coordination.

The use of dynamic functional connectivity may have some potential applications for clinical research. For example, a recent study by Lee et al. (2017) used sliding window functional connectivity when investigating the effects of anesthesia. They found that measuring the entropy of the phase locking timecourses generated throughout the experiment revealed that the complexity of frontal network connections reduced significantly fell during deeper stages of anesthesia, implying that connectivity enters a configuration where network behavior becomes more predictable. We would also like to highlight some early electrophysiological studies in the psychiatric and neurological field where a sliding window approach was successful in finding disease-induced effects. Starting with neurology, the additional value of dynamic network approaches to static network approaches has recently been demonstrated in a patient cohort undergoing resective neurosurgery (Carbo et al., 2017). Carbo and colleagues tracked participation communication of subnetworks being mediated by smaller hubs in both a static and dynamic network framework a year after surgery. They found that the dynamics of hubs in

510 occipital and default mode networks could predict whether patients had undergone a decline in verbal memory. Crucially, dynamics were key to relating network behavior to pathology, as the time-averaged hub properties were not significantly related to verbal memory impairments (Carbo et al., 2017). In another recent study on dynamic networks in epilepsy (Khambhati et al., 2017), the authors revealed similar subnetworks, using non-negative matrix factorization, in interictal and ictal data. Interestingly, by using these subnetworks obtained from interictal data, they were able to predict to some extent seizure onset regions. With respect to psychiatry, classification of patients with depression was the goal in a MEG study (Lu et al., 2013) where the authors probed dynamic effective connectivity between visual, cingulate and insula cortices during a visual task and reached a classification accuracy of $88 \%$. In an fMRI/MEG study on schizophrenia by Cetin and colleagues (2016), connectivity data were classified 520 using machine learning methods. The authors demonstrated that a dynamic connectivity approach led to a higher classification of individual subjects than a static connectivity approach. In summary, despite the fact that the sliding window approach in electrophysiological network analysis is relatively new, 
already a number of important findings have emerged regarding the nature of network connectivity and its perturbation by disease. This technique is likely to be fruitful for future research in this area. 


\section{Beyond sliding windows}

\subsection{Limitations of the sliding window}

Whilst clearly productive, the sliding window approach also has important limitations. The first issue is how to choose the window width $d$; if $d$ is too short, results are likely to be driven by noise; on the

530 contrary, long windows may fail to capture rapid changes in connectivity. Ideally, one would choose a window width that matches the timescale of the underlying fluctuations of connectivity, but these timescales are a-priori unknown and may likely vary over the course of an experiment. At least, a window width for dynamic connectivity analysis should be frequency-specific, and should depend on the number of degrees of freedom in a windowed time series, which itself depends on the frequency band of interest. Fourier theory shows that the temporal degrees of freedom of a timeseries is defined as $\eta=2 B_{w} d$, where $B_{w}$ is the spectral bandwidth of the data and $d$ is the window width. To give examples, meaningful results have been obtained in MEG/EEG data using relatively short windows ranging from $\sim 10 \mathrm{~s}$ to below $1 \mathrm{~s}^{1}$. It is important to note here that these windows are far shorter than those possible using $\mathrm{FMRI}$ data, meaning the temporal scale of connectivity fluctuations probed can be

540 much shorter. For example, if a window width of $5 \mathrm{~s}$ was applied to beta band $(13-30 \mathrm{~Hz})$ filtered data, $\eta=170$, and the uncertainty in the correlation measure would be $\sim 1 / \sqrt{ } \eta=0.08$ (Bendat and Piersol, 2011). To match the same number of degrees of freedom with $f M R I$ data, where the fastest acquisition sequences give a whole-head coverage with a sample rate of $2 \mathrm{~Hz}$, you would need a window which is $42.5 \mathrm{~s}$ wide, considerably longer. Selection of window width aside, sliding window timecourses have reduced temporal resolution compared to the underlying data. For example, if a window was 3 seconds wide and there was an instantaneous increase in connectivity between two regions at $t=0 \mathrm{~s}$, then it is possible that this event would be detected at any window centered between $-1.5 \mathrm{~s} \leq t \leq 1.5 \mathrm{~s}$. If a window is sufficiently large relative to the task it surrounds, this could obfuscate in time when a connection changed. Again, shorter window widths offered by electrophysiological data mean this

550 temporal uncertainty is much lower than what would be found with fMRI data.

\subsection{Higher temporal resolution measures of connectivity}

It is possible to dispense with the sliding window entirely in estimating the connectivity dynamics. Some connectivity metrics offer the ability to infer connectivity on an (almost) sample-by-sample level without using windows at all. For example, continuous wavelet transforms offer a computationally light estimate of instantaneous phase and power across the time series; these can be exploited to reveal coherence, partially directed coherences and Granger Causality time courses in a spectra and time-resolved manner (Dhamala et al., 2008). Applying the Hilbert transform on a wideband signal also provides an estimation of instantaneous canonical phase and amplitude per time point, which allows us to build 560 simpler methods that can exploit such high temporal resolution, such as the phase difference derivative (PDD; Breakspear et al., 2004). This metric captures similar information to conventional phase coherence (i.e. the stability of a phase difference over trials) but also quantifies its stability over time. The idea is that if a stable phase relationship exists between two time courses for a given period, then the instantaneous derivative of their phase difference is close to zero, yielding a high value of phase locking. PDD can take any value between one and zero, although it is more often used to generate binary representations of connectivity (where one refers to coupling and zero to no coupling). Whilst in principle this offers connectivity metrics on a sample-by-sample basis, usually one requires that a fixed phase relationship lasts for at least cycle of an oscillation of interest to increase robustness. PDD can be used in both rest and task. In rest, we can average multiple successive PDD metrics to generate a 570 fractional level of locking during an epoch. Alternatively, for time locked responses during a task, we can then average across trials to generate a connectivity time course at the scale of the experimental recordings (much like assessing state activations, as depicted in Figure 3 ).

\footnotetext{
${ }^{1} \mathrm{~A}$ list of the window widths utilised in all of the electrophysiological studies mentioned in this paper can be found in the supplementary material.
} 
In contrast to sliding window approaches, instantaneous measures of connectivity allow tracking of transient networks evolving at the millisecond timescale. This advantage has been exploited in neuroscientific experiments. For example, a study by Martini and colleagues (2012) on the neural basis of unpleasant picture viewing used the time-resolved phase-locking value as a connectivity metric (providing a connectivity estimate for each time point). The authors showed that unpleasant picture viewing induced stronger gamma band synchronization between interhemispheric regions of temporal

580 and frontal cortex in the early stages of the experiment (Martini et al., 2012). A dynamic reconfiguration of functional networks was observed during a picture naming task (Hassan et al., 2015) using phase lag calculations between nodes calculated for individual timepoints. Six networks were identified using k-means clustering, which showed strong phase locking at different times after the stimulus. Each of these networks became the dominant configuration at distinct periods over the space of a $500 \mathrm{~ms}$ trial, revealing the individual stages of visual processing, recognition, semantic processing and articulation. In a recent study on sensory gating (i.e. weakening of a neural response in reaction to a second identical stimulus), participants were given a paired pulse to the tibial nerve (Wiesman et al., 2017). Using a phase-locking value averaged across trials, the authors showed a sample-to-sample connectivity time course for their experiment. They showed a somatosensory gating phenomenon, where a second

590 stimulus elicits a lower amplitude oscillatory response compared to the first. Further the extent of task related phase-locking modulation is also diminished by the second stimulus compared to the first. A study by Gao and colleagues (2015) sought to investigate the dynamics of information flow during mechanosensation by using temporally-resolved partially directed coherence. They showed a dynamical reorganization of directed connectivity between the contralateral primary somatosensory cortices and bilateral secondary somatosensory cortices, characterising dynamic top-down and bottomup processes within $350 \mathrm{~ms}$ of tactile stimulation. The time dependent role of networks has also been shown in a cognitive task. A study by Cocchi and colleagues (2011) used time resolved wavelet coherence (Grinsted et al., 2004) to find fluctuations in coherence between occipital and frontal regions that were modulated during spatial perception task, which itself was embedded within the maintainance

600 period of a working memory paradigm. They found that coherence between these areas would rise and fall within the first $200 \mathrm{~ms}$ of the presentation of the spatial perception task, with the magnitude of the coherence increasing if the concurrent working memory load increased.

Some studies have also demonstrated the additional value of instantaneous measures of connectivity in the clinical context. The PDD has been applied in a study aimed to understand the neural correlates of anxiety and aggression in children. Whilst static connectivity was insensitive, they demonstrated that the PDD was sensitive to the severity of anxiety (Lackner et al., 2014). In a study analyzing resting state connectivity in a cohort of children from 0.4-16 years old, PDD showed that the length of intervals over which constant phase locking across the brain occurred grew longer with age (Thatcher et al., 2009).

610 Interestingly the same study also showed that these intervals surged in length in the subjects who were undergoing growth spurts. Another area where dynamic networks may have clinical relevance is epilepsy. It is believed that the network hubs play an important role in the generation or spread of epileptiform activity (van Diessen et al., 2013), and so dynamic connectivity can help us to understand the underlying processes. In a resting-state MEG study (Coito et al., 2015), interictal data were acquired in order to understand differences in network behavior during spiking. They showed that information flow was maximized during spiking compared to rest, with the largest drivers of information flow being the epileptogenic zones. Furthermore, they also showed in the networks detected during left and right temporal lobe epilepsy were not symmetric and topographically distinct to each other. An implication of this finding is that a single treatment approach for temporal lobe epilepsy may perhaps need to be instead separated into two drastically different treatments.

\subsection{Hidden Markov Models}

In Section 3.3, we discussed various methods to categorise multiple connectivity measurements into states to assess network behaviour. Here, we refer to an approach, the Hidden Markov Model (HMM; Baker et al., 2014; Rabiner, 1989; Vidaurre et al., 2016) that makes a direct use of the data in order to 
find a discrete set of states. The essence of such decomposition, as illustrated in Figure 4, is that, instead of estimating connectivity for each window, it performs the estimation at the state level, in such a way that all the data corresponding to that state (i.e. using all the occurrences of the state in the entire time series) is effectively pooled together to characterise the network. This advantageously avoids the need of pre-specifying the sliding window width parameter. A state itself is characterised by the spatiotemporal properties of the source level signal, such as the mean and covariance. The HMM inference, when fed with empirical data, estimates the probability of each state being active at each time point of a multivariate time series together with the probability distribution that describes each state.

Determining the optimal number of states needed for HMMs can be done in a variety of ways. A datadriven approach, if we use Bayesian inference, is to use the free energy as a model selection criterion, which balances a term for the data likelihood (i.e. how well the model describes the data) with a term for the model complexity. Alternatively, non-parametric approaches can estimate the optimal number of states directly (Beal et al., 2002). In a neuroscience context, however, manual corroboration of the 640 results in conjunction with automatic optimisation is recommended to ensure the states generated are at least interpretable. A notable challenge is how to scale the HMM inference up to work on very large numbers of time-points and/or subjects (e.g. many hundreds of subjects). Recent developments have shown that this can be achieved using approaches based on stochastic learning (Vidaurre et al., 2017a).

The probability distribution describing each state is referred to as the observation model; different choices for the observation model probability distribution are possible, such that we can tailor the choice of this probability distribution to the particularities of the data modality at hand. For instance, Baker and colleagues (2014) modelled the power envelope of resting-state MEG data across the whole brain using a Gaussian observation model HMM. This version of the HMM describes each state using a mean 650 (describing the average amplitude activation when the state is active) and a covariance matrix (describing the functional connectivity in terms of amplitude correlations). As a result of this analysis, it was shown that the classical 'resting state networks' rapidly form and dissolve with lifetimes averaging between 100-200 ms. A limitation of this approach is that it cannot model the phase information of the raw data. As a solution, Vidaurre et al. (2016) proposed a different version of the HMM where the states are represented by multivariate autoregressive models (HMM-MAR) acting on the raw time series instead of on the power envelopes, and thus, having access to phase information. Therefore, the states are described not only by power but also by phase coupling, acknowledging the specific phase differences between each pair of regions. Using this model, the data get spatially, temporally and (considering that these features are given as a function of frequency) spectrally resolved. As a proof of concept, the HMM-MAR was shown to characterise fast spectral changes in between the primary somatosensory areas in a simple button press experiment (Vidaurre et al., 2016). In this work, dynamic connectivity aspects of the movement-related beta synchronisation and desynchronisation responses were analysed, allowing to link typical time frequency spectrograms to connectivity modulations. A limitation of this method is its relative sensitivity to the choice of the MAR order parameter; despite existing data-driven ways to guide this choice, a too high order can easily overfit the data when the number of regions is large. Hence, given that the number of parameters scales quadratically with the number of regions, the HMM-MAR is typically more practical for modelling a few regions at a time.

When confronted with whole-brain data, however, one possible alternative to reduce the model complexity is to dispense with the cross-region terms of the MAR model. The states would then be defined in terms of spectrally-defined power changes but not by connectivity. Another possibility is to use a novel version of the HMM based on a time-embedded transformation of the data combined with a simple Gaussian observation model; this can identify states with particular autocorrelation and nonzero lag cross-correlations that approximate the state-specific power spectra and phase locking information accessible with the HMM-MAR, but using a less complex model and, therefore, bypassing possible overfitting issues (Vidaurre et al., 2017b). This approach has been used in whole-brain restingstate MEG data, revealing frequency specific phase-locking networks much more clearly than is 
possible using a static perspective. Further, this reveals an interesting fragmentation of the DMN into two spectrally well-defined components: an anterior theta/delta component with strong connectivity with the posterior cingulate cortex, and an alpha posterior component including parietal and temporal regions.
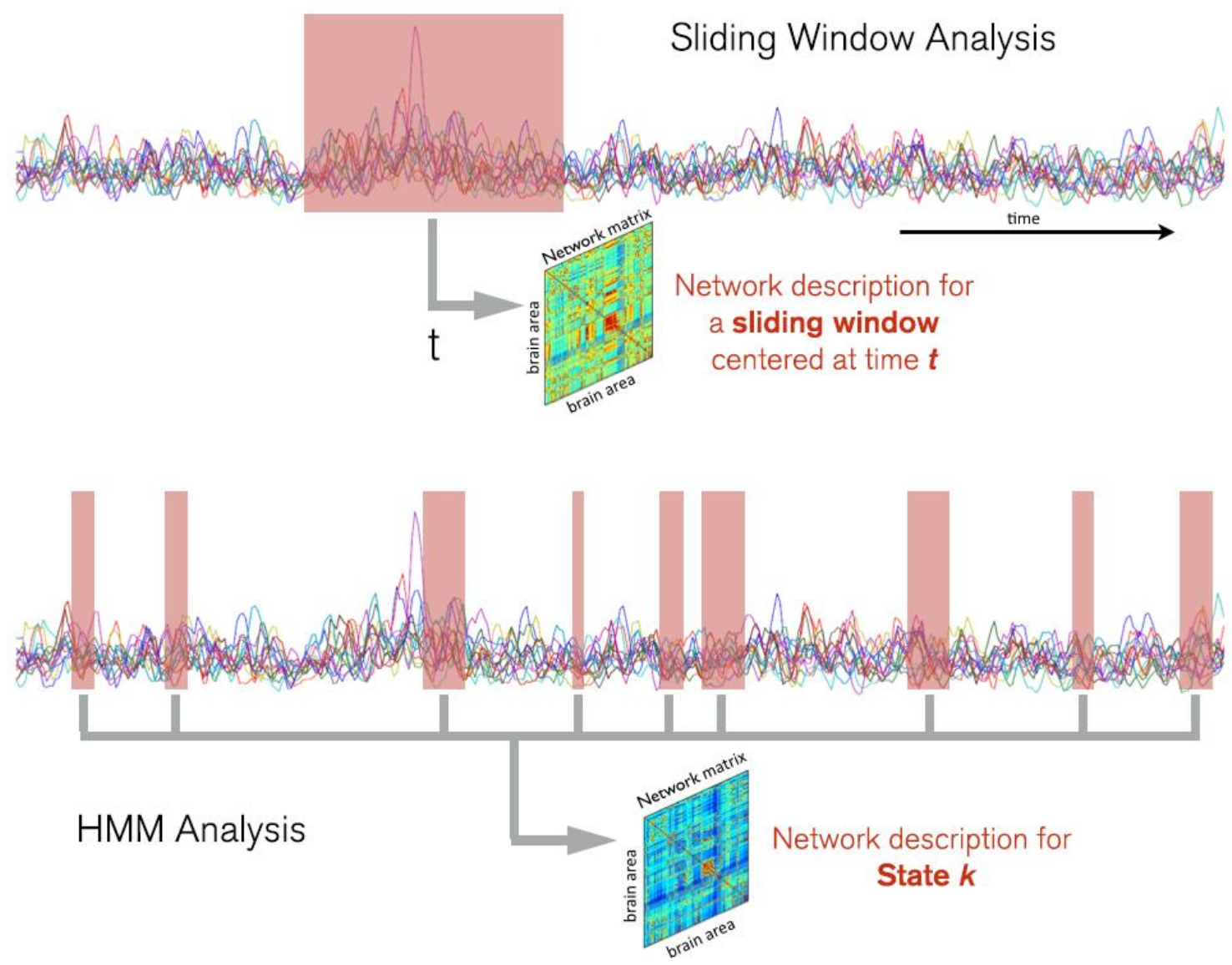

Figure 4: Hidden Markov Modelling (HMM) network analysis (bottom) as opposed to sliding-window network analysis (top). Whereas the sliding window has a fixed width and ignores the data beyond its boundaries, the HMM automatically finds, across the entire data set, all the network occurrences that correspond to a given state, enhancing the robustness of the estimation (because it has more data than a window) and adapting to inherent network time in a data driven manner. In this example, the states themselves reflect unique spatial patterns of oscillatory envelopes and envelope couplings, that consistently repeat and different points in time. The non-marked segments of the data correspond to other states.

\subsection{Future Developments}

A common assumption with many of the methods and studies covered in this review is that connectivity is assessed within a single frequency band. Given what we know of the complex spectral dynamics of electrophysiological oscillations, it stands to reason that we are failing to capture many of these connections. Multilayer network analysis (Boccaletti et al., 2014; Kivelä et al., 2014) provides a framework to allow for not only multiple within-band networks (captured as separate 'layers', as shown in Figure 5), but also cross-frequency coupling, which is modelled as between layer connections. This framework has been applied in the stationary connectivity domain (Brookes et al., 2016; Tewarie et al., 2016), where it has been shown to find connectivity abnormalities in an occipital network in schizophrenia which scale with the severity of (negative) patient symptoms (Brookes et al 2016). In principle, it could be used in conjunction with a dynamic framework to generate time-evolving multilayer networks. In contrast to multilayer networks in the static domain, the additional assumption with respect to temporal networks is that the ordering of the layers in the multilayer network contains information 
(Holme and Saramaki, 2012). A special case of multilayer networks, multiplex networks assume oneto-one between-layer connections, and so it is possible to ignore inter-layer coupling. In a dynamic approach this would treat each time point of a network as individual layers. In this formulation, one could make use of the range of graph or network measures available to assess on temporal networks and 710 graphs (Cozzo et al., 2016; Nicosia et al., 2013). Neuroimaging-focused toolboxes to analyse data in this way have recently been made available (Sizemore and Bassett, 2017), which include extended versions of graph theoretic metrics such as centrality, community structure, clustering and path length. However, a challenge that future studies will need to address before dynamic graph analysis can be employed at large is how to compare time varying functional networks between groups, without the bias of link density, network degree and average connectivity (Tewarie et al., 2015; van den Heuvel et al., 2017; van Wijk et al., 2010).

Whilst expanding analysis to incorporate multiple frequency bands of neuronal oscillations is an improvement over a single band, it can be argued that this is still too simplistic picture to characterize 720 the electrophysiological connectome. Rather than seeing the brain as either an ordered (oscillatory) system or a random (disordered) system, we can alternatively use the entire broadband signal and treat the brain as a critical system (Haimovici et al., 2013) which straddles these two regimes. Critical systems are scale-free, meaning their behaviour can be characterized using power laws. Extracellular electrophysiological systems and their fluctuations in power have all been shown to follow power laws (He et al., 2010; Linkenkaer-Hansen et al., 2001; Miller et al., 2009; Poil et al., 2008), as does global functional connectivity (Botcharova et al., 2014; Ton et al., 2015). Furthermore, a recent study by Wen and Liu (2016) showed that fluctuations in EEG broadband power would better predict how the global resting state fMRI signal would modulate better than band limited power timecourses. Specifically, global hemodynamic changes would lag approximately 5 seconds behind broad band power changes,

730 implying scale-free dynamics are a contributor to fluctuations seen in resting state networks. Recently, this combined approach of power law analysis was applied to fluctuations in phase synchronization in the clinical context of autistic children and children with reading difficulties (Dimitriadis et al., 2013; Tinker and Velazquez, 2014). Future studies should evaluate the use of this type of analysis not merely on bivariate or global synchronization, but on the formation and dissolution of networks, and whether spread of large scale activity and connectivity is also scale-invariant (Poil et al., 2012).

Given that the fMRI community has also poured significant time into the field of dynamic functional connectivity (Hutchison et al., 2013; Preti et al., 2016), it is worth mentioning a few approaches from $\mathrm{fMRI}$, which may be compatible but have not necessarily been applied to electrophysiological data. If

740 the connectivity community continue to use sliding windows, we may be able to use the data to dynamically adjust the width of the sliding window, rather than assuming the width must be fixed, i.e. a data-driven window selection. Applications of this approach in the field of electrophysiology are scarce, but an example from the fMRI community is the dynamic connectivity detection approach (Xu and Lindquist, 2015). This approach seeks separable partitions in multivariate time courses, for which the mean and covariance are significantly different to neighbouring partitions. This approach has been applied in a facial recognition MEG study in depression. The authors employed their approach in combination with machine learning (support vector machines), and were able to classify patients with depression from healthy controls with an $83 \%$ accuracy (Bi et al., 2016). If one assumes that connectivity dynamics are characterised by short periods of network synchronisation alternated with periods of desynchronisation, one could just focus on detecting these transient periods of strong synchronisation. A method targeting these transient periods is point process analysis (Tagliazucchi et al., 2016; Tagliazucchi et al., 2012). Here, only data points for which the signal amplitudes cross a threshold are used for network analysis. Although this method uses only amplitude information to find the data points for which connectivity will be estimated, theoretical work suggests that amplitude and connectivity modulations are, often, closely related (Daffertshofer and van Wijk, 2011; Moon et al., 2015). One final alternative approach to the sliding is the dynamic conditional correlation (DCC) model (Lindquist et al., 2014), which has been applied to fMRI. By calculating correlation between timeseries 
as a function of their past correlations and standard deviations, this autoregressive-based model was able to detect changes in the correlations/covariance that were not induced by random noise. The particular strength of DCC is that in a null simulation (where there were no correlations modelled between the seed and test timeseries) DCC managed to closely follow the null where sliding windows would show highly fluctuating correlations.

\section{Spectral Multilayer Network}

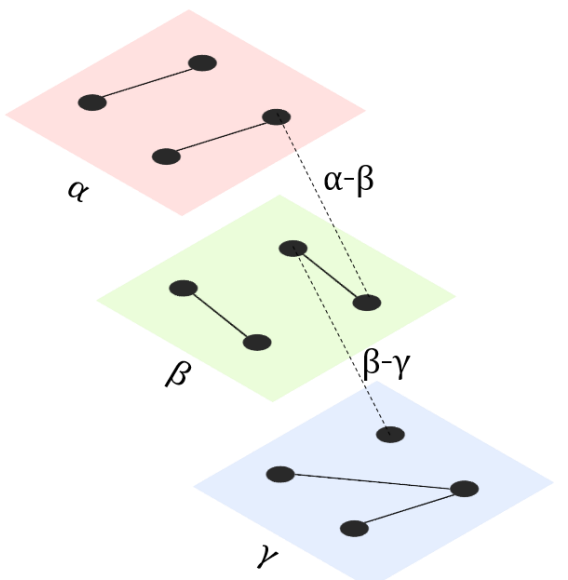

Temporal Multiplex Network

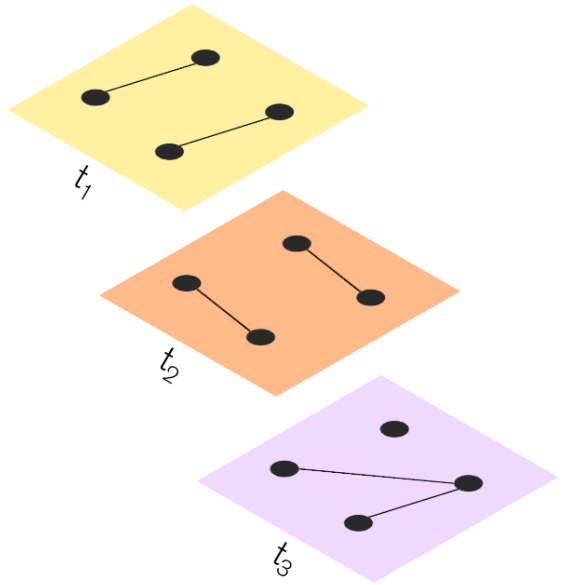

FIGURE 5: Example networks which may be applied into future studies of dynamic functional connectivity. LEFT: A Multilayer network, where each layer represents a different frequency band of neural oscillations. Within band connections are represented as solid black lines between nodes on the same layer, cross frequency relations are represented as dashed lines between layers. RIGHT: A special case of a multilayer network, where links between layers are ignored; this is known as a multiplex network. In this example, each time point is modelled as individual layer. 


\section{Models for dynamic electrophysiological networks}

\subsection{The need for computational modelling}

In basic sciences such as physics and chemistry, advances in knowledge are achieved by a continuous interaction between experimental findings and quantitative theories. Quantitative theories allow for understanding of a system of interest and enable us to make mechanistic predictions, e.g. the laws of classical mechanics allows one to predict the trajectory of a projectile in orbit given certain initial conditions. The interaction between quantitative predictions of a theory and experiments allows one to assess the error of the theoretical predictions. This interplay enables falsification, verification, or refinements of theories and has led to revolutions in the sciences, of which relativity is a good example.

In the field of neuroimaging, quantitative theories are largely lacking, which prevents us from making any predictions of connectivity that can be verified using experiments. This hampers the advance in knowledge and understanding of the underlying mechanisms that drive modulations in connectivity. Importantly, due to a lack of quantitative theories we regularly have no clear explanation for experimental findings of connectivity. For example, in the clinical field, increases in connectivity in certain regions in a diseased group are often interpreted as compensatory effects, or as functional

790 reorganisation (Schoonheim et al., 2015). However, increases in connectivity in diseased groups could just as easily be explained by disinhibition induced by pathology. The reason for changes of connectivity could lie in the duration and/or reoccurrence of the periods of synchronisation versus desynchronization (Stam et al., 2005). Therefore, elucidating the mechanisms that drive connectivity fluctuations will help the clinical field to understand these disease-induced alterations in connectivity. In addition, connectivity estimates in diseased populations are often correlated to structural MRI measures, such as atrophy or white matter integrity. The problem with these associations quantified from empirical data is that we cannot infer causality, whereas, quantitative theories could aid us in the context of causal relationships. Finally, a fundamental insight into the driving factors of connectivity fluctuations is also important from a purely neuroscientific perspective. This research has the potential of bridging the gap between 800 microscopic and macroscopic activity and connectivity (Breakspear and Stam, 2005). Taken together, the field has to come to an understanding of the factors that drive modulations in connectivity in order to adequately interpret their experimental results.

\subsection{Available models}

We refer to Box 1 for the definition of some basic concepts that are used in this section. Computational models that have been used in the context of large-scale functional networks can be divided into biologically informed models and non-biologically informed models. In the context of biologically informed models, we could treat the brain as a large set of single cell neurons covering the entire brain and wired using white matter tract information from diffusion tensor imaging (DTI). However, such a

810 large system can easily become unstable, and it is difficult to infer underlying principles in a system with such an immense parameter space (Izhikevich and Edelman, 2008). This complexity can be mitigated by, for example, studying connectivity between two neuronal populations. This has the advantage of studying a system with clear underlying biophysics and neurobiological realism (Jones et al., 2000). Yet, most studies on large-scale electrophysiology employ reduced models, such as neural mass or neural field models (Coombes et al., 2014a; Coombes et al., 2014b; Deco et al., 2008), which are more tractable and at the same time support various types of behaviour. Neural mass models come in various forms, they describe the mean activity of a distinct neuronal population, typically ignoring the variance of the activity. However, variance can be incorporated into an extended model such as in Buice et al. (2010). These neural mass models are usually formulated in terms of ordinary differential equations;

820 thus, their state variables only vary as a function of time and not as a function of space. Spatial information at the network scale is usually modeled by sampling a set of neural masses over the cortex and connecting them through DTI-based tracts. One can subsequently decide whether or not to include axonal conduction delays for connections in the network (Deco et al., 2009). Neural fields on the other hand are formulated in terms of partial differential equations or in integro-differential equations and thus naturally incorporate space. These models usually treat the cortex as a 2D continuous sheet and can 
support various spatiotemporal periodic patterns, travelling waves and standing waves (Coombes, 2005). Some partial differential equation neural field models can be reduced to neural mass like models by, for example, setting the Laplace operator to zero (Hindriks and van Putten, 2013; Robinson et al., 2002).

830

In contrast to biologically informed models, one could also opt to use non-biologically informed models to study large-scale functional networks. The rationale for using non-biologically informed models for large scale networks is that at a phase transition for global connectivity, the details of the model become less relevant (Haimovici et al., 2013). Examples are the Kuramoto model (Breakspear et al., 2010), coupled Stuart-Landau oscillators (Deco et al., 2017), linear threshold models (Misic et al., 2015; O'Dea et al., 2013), network diffusion models (Abdelnour et al., 2014) and epidemic spreading models (Meier et al., 2017; Stam et al., 2016). The Kuramoto model of coupled phase oscillators is used to study network synchrony. The original model is based on an all-to-all connectivity pattern, which can be updated in the context of brain networks to empirically determined DTI networks. Stuart-Landau oscillators are oscillators whose working point can be tuned around a Hopf-bifurcation (see Box 1). Similar to neural mass models, these oscillators can be studied in a network context by allowing the oscillators to interact using a structural network derived using DTI (Deco et al., 2017). Network diffusion models and epidemic spreading models were developed in the field of complex networks. This type of interaction models has recently been applied to predict MEG and fMRI network patterns from the underlying structural network (Abdelnour et al., 2014; Stam et al., 2016; Meier et al., 2017). The network diffusion model simulates diffusion on a network. The equations are very similar to the heat diffusion equation, but the Laplace operator in the diffusion equation is translated into the graph Laplacian Epidemic spreading models are a class of models that were originally designed simulate epidemic spreading over a population. A well-known example is the susceptible-infected-susceptible model (SIS): nodes in this network can either be infected (active) or susceptible (inactive, but potentially excited by its neighbours). This model has recently been used to analyse how effective connectivity patterns with a posterior-anterior gradient in the alpha band, measured using transfer entropy from resting-state MEG data, emerge from the topology of the structural network (Meier et al., 2017).

\subsection{Insights from computational models}

The first modelling studies on electrophysiology aimed to understand the particular nature and characteristics of bivariate synchronisation that are observed in EEG signals (Breakspear, 2004). Special interest was placed in understanding the existence of nonlinear synchronisation in human EEG data (Breakspear, 2004; Breakspear and Terry, 2002a; Le Van Quyen et al., 1999; Stam et al., 2003).

860 Modelling studies showed that, by tuning a global coupling parameter in a network of conductancebased neuronal oscillators, one can switch between a regime with independent oscillations to a regime with chaotic synchronization, between which a weakly stable state exists that is considered consistent with empirically observed synchronization (Breakspear and Terry, 2002b; Breakspear et al., 2003). Largely due to the appearance of numerous fMRI-based connectivity studies, the modelling field switched to analysing large-scale static fMRI networks (Breakspear, 2017; Deco et al., 2011), and, more recently to large-scale dynamic fMRI networks (Hansen et al., 2015; Ponce-Alvarez et al., 2015).

Although we refer the reader to recently published reviews on dynamical models applied to fMRI based networks (Breakspear, 2017; Deco et al, 2011) for more comprehensive descriptions, we briefly mention

870 some of the working hypotheses that are relevant to the electrophysiological literature. Concepts from nonlinear dynamics and complex network theory have both been used to elucidate how functional networks emerge from the underlying anatomical wiring (Goñi et al., 2014; Meier et al., 2016; Robinson et al., 2014). One hypothesis is that empirically observed static functional networks are explained by models that include noise and whose working point is near a bifurcation, which separates a linear regime and a multi-stable regime (i.e. presence of multi-stable attractors, or states; see Box 1), and where the role of noise is to allow for switching between attractors (Deco and Jirsa, 2012). The repertoire of these converging brain states or attractors is considered to partly emerge from the topology of the anatomical 
network, for example, rich-club organisation supports diversity of attractors (Senden et al., 2014). Apart from multistability, models with metastable working points are also consistent with empirically observed 880 functional connectivity (Breakspear, 2002). Unfortunately, these explanatory studies for static largescale electrophysiological networks have been few and far between. The first studies on static MEG networks were based on simulations of alpha band activity (Nakagawa et al., 2014; Tewarie et al., 2014). A spiking-neuronal network showed that the inclusion of axonal conduction delays significantly improved the fit to empirical MEG networks, based on amplitude envelope correlations (Nakagawa et al., 2014). Another modeling study on static MEG networks employed a network of neural masses. They demonstrated that the structural degree product of the underlying structural network, and an operating point for global coupling strength near a phase transition for synchrony allowed for simulation of phase based network patterns similar to empirically observed alpha band networks (Tewarie et al., 2014).

890 Complex network studies have also helped in elucidating how static functional electrophysiological networks emerge from structure, explaining the presence of strong functional connections in the absence of a direct structural link (Goñi et al., 2014; Saggio et al., 2016; Stam et al., 2016; Tewarie et al., 2014). These explanations include polysynaptic walks in the structural network that contribute to electrophysiological connectivity strength (Meier et al., 2016; Robinson, 2012), and, relatedly, the contribution of detours along the shortest paths in the structural network (Goñi et al., 2014). However, when analysing shorter time scales, these structure-function mappings based on the weighted adjacency matrices fail (Honey et al., 2007; Ton et al., 2014), and, as a consequence, we need to rely on realistic computational models to explain time varying connectivity patterns. The first study in the context of dynamic MEG networks showed that the standard deviation of broadband envelope correlations could be explained by metastability induced by delayed interactions between nodes in a Kuramoto model (Cabral et al., 2014; see Figure 6). If electrophysiological networks are characterised by the formation and dissolution of networks or states, future computational studies will also need to investigate whether the formation and dissolution of empirically observed networks can emerge in neuronal models from merely multistability or metastability, or whether additional constraints are required. Multistability or metastability as working points will very likely show time-varying networks, but it remains an open question as to whether these simulated time-varying networks emerge with the same spatiotemporal signatures as we observe in empirical electrophysiological data (for example as found by Hidden Markov Model approaches). A recent theoretical study introduced a new mathematical framework based on the Kuramoto model that can potentially explain time-varying patterns of synchronisation observed in empirical electrophysiological data (Petkoski et al., 2016). 


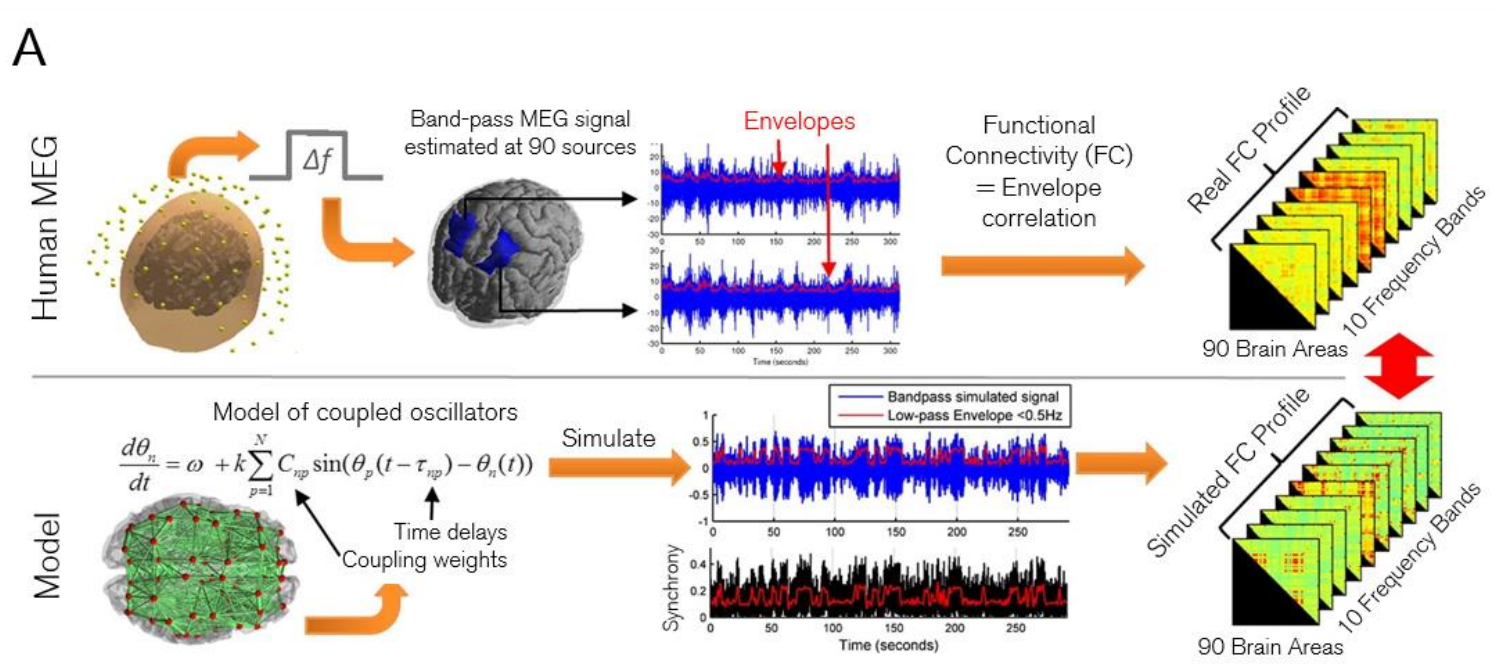

B

C
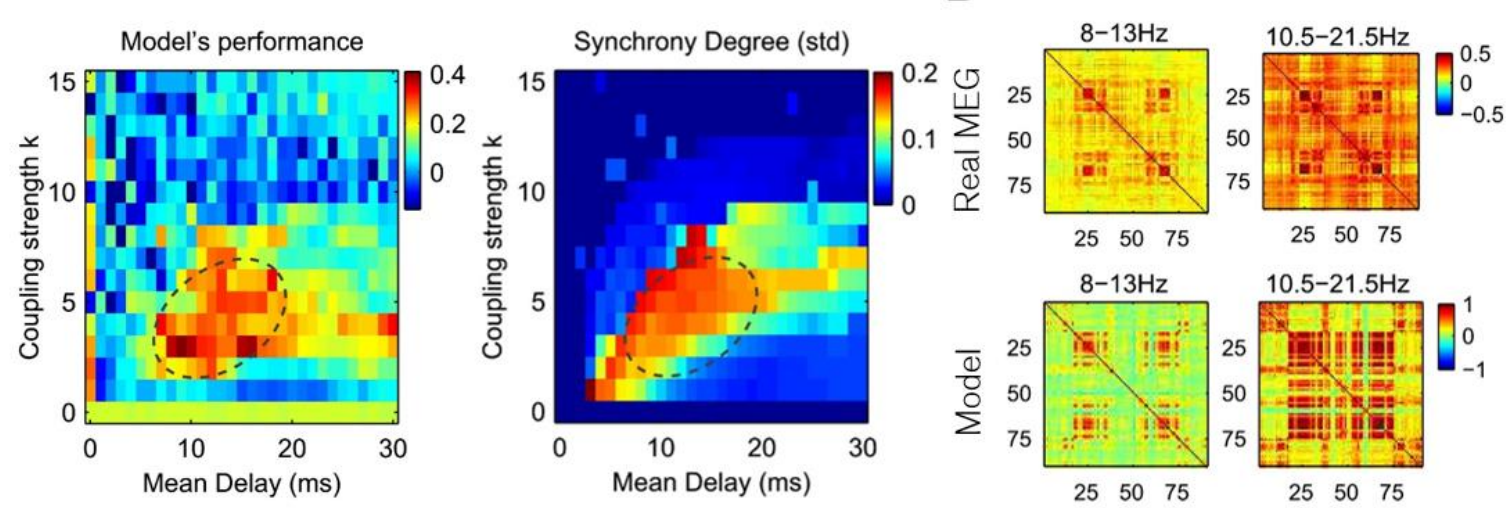

Figure 6. An example study on computational modelling and how it aids the understanding of empirical findings, in this case, comparing amplitude envelope correlations from real MEG data to those generated by coupled Kuramoto oscillators. A) The pipelines to generate the functional connectivity results based on MEG recordings (top row) and computational models (bottom). B) The correlation between the empirical data and model, revealing that the model bears strongest resemblance to the empirical findings when temporal delays of $\sim 16 \mathrm{~ms}$ are incorporated into the model with intermediate coupling strengths (dashed oval region). C) Standard deviation of the model's intrinsic synchrony over time, the high standard deviation in the oval region implies that when the model is metastable (or when synchrony strongly fluctuates over time) it best reflects real connectomes. D) Example connectome matrices from real data and a model set with a mean delay of $16 \mathrm{~ms}$ and a coupling parameter of 3 . Figure adapted with permission from Cabral et al., (2014).

\subsection{Future directions for computational models in electrophysiology}

An important point for future modelling studies on dynamic electrophysiological connectivity is model selection. A recent study in the fMRI literature compared different models to estimate empirical and static functional networks (Messe et al., 2015). Their most simple model, an autoregressive model, outperformed most of the biologically informed models (spiking models, neural mass models) and the Kuramoto model. This raised the question of whether details of the local dynamics are at all relevant at the larger scale and whether macroscopic functional networks can merely emerge from the topology of the structural network. Non-biologically informed models, such as network diffusion models and epidemic spreading models have been successfully used in this context (Abdelnour et al., 2014; Meier et al., 2017; Misic et al., 2015; Stam et al., 2016). However, a recent study on dynamic fMRI networks showed that more simple models, e.g. autoregressive models, could not explain the necessary switching between network configurations as shown in empirical data (Hansen et al., 2015). Furthermore, epidemic spreading models do not support various network configurations once the model 
reaches its quasi-static state and normal operating point (Barrat et al., 2008; Van Mieghem, 2009). In the context of dynamic electrophysiological connectivity, models are required to allow for switching between network configurations. This would therefore advocate the use of models that support metastability or multi-stability.

Future neuronal models will not only have to match spatial and temporal features of ongoing empirical connectivity modulations but also spectral properties of MEG/EEG oscillations, or even arrhythmic scale free brain activity $(\mathrm{He}, 2014)$. Most models are tuned to generate oscillations with a dominant frequency (usually alpha), whilst some models can mimic the $1 / f$ power spectrum observed in empirical data (Linden et al., 2010; Robinson et al., 2001; Stefanescu and Jirsa, 2008). In order to understand ongoing neuronal connectivity modulations, we require sophisticated models that can not only produce a wealth 950 of simultaneous frequencies, but also frequency specific networks. A recent study was able to simulate multiple (independent) network layers of Stuart-Landau oscillators, where each layer had a different dominant frequency (Deco et al., 2017). The best fit with MEG networks was achieved when the operating point for local dynamics was found near a supercritical Hopf-bifurcation (a bifurcation from a stable equilibrium to a limit cycle). As an extension to this type of approach, we also require models that can simulate frequency band specific spatial network patterns, and coupling between layers that can potentially induce phenomena such as nesting of fast oscillations to generate slower rhythms and local phase-amplitude coupling. Worth mentioning in the context of neuronal models are the next generation neural mass models (Byrne et al., 2017; Coombes and Byrne, 2016), which may prove useful in furthering our understanding of within population synchrony modulations during cognitive tasks, which

960 is important in the context of oscillations. Furthermore, these models are based on an exact mean-field description of the underlying spiking neurodynamics, which make them an excellent candidate for simulations of large scale electrophysiological networks.

One of the criticisms of the use of neuronal models to explain connectivity is their extensive parameter space and the difficulty to treat these models analytically. Whilst there are data-driven solutions to tuning these models (Freestone et al., 2011), the vast parameter space has prompted the use of more simple models of phase oscillators, such as the Kuramoto model. Despite fruitful applications of the Kuramoto model in relation to functional networks (Breakspear et al., 2010; Cabral et al., 2014), its phase interaction function (simply a sinusoid) lacks neurobiological realism. An elegant way to maintain a 970 tractable model is to use a biologically informed phase interaction function derived from any neural mass model (see Ashwin et al., 2016 for mathematical details). For example, Kuramoto-type phase oscillators can also be derived from a network of weakly coupled neural mass models, giving rise to a non-sinusoidal interaction function (Hlinka and Coombes, 2012). These model-specific phase interaction functions are derived from the phase response curves of the model and can be written in terms of a Fourier series. Most of these phase interaction functions require an operating point in the weak coupling regime (i.e. a perturbation that causes the trajectory to jump not far from the intrinsic limit cycle). However, in the case of strong coupling (e.g. during a task), it is not yet entirely what is the best approach to model these interactions.

980 In summary, advances in the development of computational modelling have been made to start to replicate the empirical findings seen in functional connectivity analysis. However, we are still at an early stage of development, where robust testing of these models is still required and ongoing. Here, the link between the empirical and modelled fields at this point becomes the most apparent; to confirm or refute the feasibility of these models, we need more experimental observations to test them with. It is then we shall be able to make predictions from models which can specify future experiments. 
Box 1: models for dynamic connectivity, concepts related to nonlinear dynamics and complex networks.

Chaos: a branch of mathematics concerned with the characterisation of dynamical systems which typically are highly sensitive to their initial conditions.

Bifurcation: the value of certain parameter of interest for which a small change brings a dramatic change in qualitative behavior of the system.

Phase space: an n-dimensional geometric space, where $n$ corresponds to the number of state variables, encompassing all possible states.

Attractors: subspace in phase space to which a trajectory converges for certain initial conditions.

Limit-cycle: an isolated periodic attractor (oscillator), which is a solution for a dynamical system which repeats itself in time.

Saddle node bifurcation: by tuning a bifurcation parameter of interest, an unstable fixed point approaches a stable fixed point until they collide and annihilate.

Hopf-bifurcation: bifurcation for which a fixed point loses stability in favour of a limit-cycle oscillation.

Multistability: co-existence of multiple attractors.

Metastability: Metastability refers to the dwelling tendency of trajectories in phase space without convergence to an attractor (or remnants of attractors). This type of dynamics can be induced by the presence of saddle.

Phase interaction function: periodic function in a network of phase oscillators that determines the phase difference coupling between oscillators. The simplest interaction function is the sine function used in the Kuramoto model (see e.g. Ashwin et al., 2016).

Phase response curves: transient change in the cycle of an oscillator described in terms of its phase due to a small external perturbation.

Rich-club: tendency of high degree nodes to be more connected to each other than to low degree nodes.

Walks: routes in a network or graph between two given nodes, which is described as a sequence of links to traverse (walks can include loops). 


\section{Summary}

In this review, we have covered the methods and applications of measuring the dynamics of large scale electrophysiological functional networks using both MEG and EEG. We have laid out the motivation for such methods, showing that other non-invasive neuroimaging methods simply cannot reach the temporal scales at which neural oscillations (and therefore cortico-cortical communication) modulate. Whilst still a nascent field of research, we have shown that there is a vast array of methods to capture the temporal dynamics of connectivity (either using sliding window or other instantaneous approaches). These methods have allowed us to push the temporal scale of functional connectivity dynamics from 1000 hours and minutes, to only a few seconds and even milliseconds, allowing us to now probe neurophysiological effects which were previously unattainable.

Functional network structures exist across a continuum of temporal scales ranging from many hours of data to just a few hundred milliseconds. Dynamic electrophysiological connectivity has shown that such networks, and the connectivities that define them, modulate not only temporally, but also spectrally and spatially. This therefore offers new dimensions in which to probe network structure and how the brain continually forms and dissolves a hierarchy of functional networks to support ongoing task demand. It is hoped that by probing connectivity at these scales, the neuroscientific insights offered will allow us to gain a better understating of mechanisms of functional communication in both health and disease. Early

1010 studies into the differences of dynamic networks between healthy and clinical populations are starting to emerge, supporting this notion. Nevertheless, in order to be clinically useful, dynamic network approaches need to meet at least two requirements: 1) To be more sensitive in identifying diseaseinduced effects than static connectivity approaches and correlate better with disease status; 2) To be more reproducible than static network approaches (Liuzzi et al., 2016; Schoonheim et al., 2015; Tijms et al., 2013). It is on these grounds that we will be able to explore the role of dynamic networks in the context of personalised prediction of disease progression and treatment response.

For us to truly understand how these networks function, and fail in pathology, we cannot merely rely on empirical findings alone. In part, the growing number of methods developed to track connectivity means we may start to see conflicting results depending on which processing pipeline is applied; for this we need a system to adjudicate these observations. Also, even if some consensus is reached on the best way to capture connectivity dynamics, these empirical results can only tell what is happening in the brain in on a macroscopic, and somewhat abstract level. To understand the underlying neurobiology, we need to apply computational models of neural dynamics to mimic what we are observing. These models offer us the potential to explain the mechanisms of communication in the human brain, as well as predictions on what we will find next. As these empirical and modelling studies continue to mature over time, we should see the gulf between connectivity and its physical basis narrow.

\section{ACKNOWLEDGMENTS}

1030 This work was funded by a Medical Research Council New Investigator Research Grant (MR/M006301/1). We also acknowledge Medical Research Council Partnership Grant (MR/K005464/1). The authors would also like thank Stephen Coombes from the School of Mathematical Sciences, University of Nottingham, for his helpful insights into computational modelling.

Box 2: Questions for future developments

- Are dynamic connectivity approaches robust and reliable enough to track dynamic networks over several cognitive experiments? Is the low SNR of encephalography a limiting factor?

- Will dynamic connectivity investigations (both empirical and computational) tell us anything

$1040 \quad$ new about the brain we didn't know before?

- What are the macroscopic, microscopic and scale-free properties at play which generate connectivity dynamics? 


\section{References}

Abdelnour, F., Voss, H.U., Raj, A., 2014. Network diffusion accurately models the relationship between structural and functional brain connectivity networks. Neuroimage 90, 335-347.

Allen, E.A., Damaraju, E., Plis, S.M., Erhardt, E.B., Eichele, T., Calhoun, V.D., 2014. Tracking whole-brain connectivity dynamics in the resting state. Cereb Cortex 24, 663-676.

Ashwin, P., Coombes, S., Nicks, R., 2016. Mathematical Frameworks for Oscillatory Network Dynamics in Neuroscience. J Math Neurosci 6, 2.

Baccala, L.A., Sameshima, K., 2001. Partial directed coherence: a new concept in neural structure determination. Biological Cybernetics 84, 463-474.

Baker, A.P., Brookes, M.J., Rezek, I.A., Smith, S.M., Behrens, T., Probert Smith, P.J., Woolrich, M., 2014. Fast transient networks in spontaneous human brain activity. Elife 3, e01867.

1060 Baker, A.P., Luckhoo, H., Brookes, M.J., Smith, P., Woolrich, M., 2012. Investigating the Temporal Dynamics of Resting State Brain Connectivity using Magnetoencephalography., Organization of Human Brain Mapping. , Beijing, China.

Barrat, A., Barthelemy, M., Vespignani, A., 2008. Dynamical processes on complex networks. Cambridge university press.

Bassett, D.S., Porter, M.A., Wymbs, N.F., Grafton, S.T., Carlson, J.M., Mucha, P.J., 2013. Robust detection of dynamic community structure in networks. Chaos 23.

Bastos, A.M., Schoffelen, J.M., 2015. A Tutorial Review of Functional Connectivity Analysis Methods and Their Interpretational Pitfalls. Front Syst Neurosci 9, 175.

1070

Beal, M.J., Ghahramani, Z., Rasmussen, C.E., 2002. The infinite hidden Markov model. Advances in neural information processing systems, pp. 577-584.

Beckmann, C.F., DeLuca, M., Devlin, J.T., Smith, S.M., 2005. Investigations into resting-state connectivity using independent component analysis. Philosophical Transactions of the Royal Society B-Biological Sciences 360, 1001-1013.

Bendat, J.S., Piersol, A.G., 2011. Random data: analysis and measurement procedures. John Wiley \& Sons.

Berger, H., 1929. Electroencephalogram in humans. Archiv Fur Psychiatrie Und Nervenkrankheiten 87, 527-570.

Betti, V., Della Penna, S., de Pasquale, F., Mantini, D., Marzetti, L., Romanis, G.L., Corbetta, M., 2013. Natural Scenes Viewing Alters the Dynamics of Functional Connectivity in the Human Brain. Neuron 79, 782-797.

Betzel, R.F., Fukushima, M., He, Y., Zuo, X.N., Sporns, O., 2016. Dynamic fluctuations coincide with periods of high and low modularity in resting-state functional brain networks. Neuroimage 127, 287-297.

Bi, K., Hua, L., Wei, M., Qin, J., Lu, Q., Yao, Z., 2016. Dynamic functional-structural coupling within acute functional state change phases: Evidence from a depression recognition study. J Affect Disord 191, 145-155.

1090 Biswal, B., Yetkin, F.Z., Haughton, V.M., Hyde, J.S., 1995. Functional connectivity in the motor cortex of resting human brain using echo-planar MRI. Magn Reson Med 34, 537-541.

Boccaletti, S., Bianconi, G., Criado, R., del Genio, C.I., Gomez-Gardenes, J., Romance, M., Sendina-Nadal, I., Wang, Z., Zanin, M., 2014. The structure and dynamics of multilayer networks. Physics Reports-Review Section of Physics Letters 544, 1-122.

Botcharova, M., Farmer, S.F., Berthouze, L., 2014. Markers of criticality in phase synchronization. Front Syst Neurosci 8, 176.

Breakspear, M., 2002. Nonlinear phase desynchronization in human electroencephalographic data. Hum Brain Mapp 15, $175-$ 198.

Breakspear, M., 2004. "Dynamic" connectivity in neural systems: theoretical and empirical considerations. Neuroinformatics 2 , 205-226.

Breakspear, M., 2017. Dynamic models of large-scale brain activity. Nat Neurosci 20, 340-352.

Breakspear, M., Heitmann, S., Daffertshofer, A., 2010. Generative models of cortical oscillations: neurobiological implications of the kuramoto model. Front Hum Neurosci 4, 190.

Breakspear, M., Stam, C.J., 2005. Dynamics of a neural system with a multiscale architecture. Philos Trans R Soc Lond B Biol Sci 360, 1051-1074

Breakspear, M., Terry, J.R., 2002a. Detection and description of non-linear interdependence in normal multichannel human EEG data. Clin Neurophysiol 113, 735-753.

Breakspear, M., Terry, J.R., 2002b. Nonlinear interdependence in neural systems: motivation, theory, and relevance. Int J Neurosci 112, 1263-1284. 
Breakspear, M., Terry, J.R., Friston, K.J., 2003. Modulation of excitatory synaptic coupling facilitates synchronization and complex dynamics in a biophysical model of neuronal dynamics. Network 14, 703-732.

Breakspear, M., Williams, L.M., Stam, C.J., 2004. A novel method for the topographic analysis of neural activity reveals formation and dissolution of 'Dynamic Cell Assemblies'. J Comput Neurosci 16, 49-68.

Brookes, M.J., Hale, J.R., Zumer, J.M., Stevenson, C.M., Francis, S.T., Barnes, G.R., Owen, J.P., Morris, P.G., Nagarajan, S.S., 2011a. Measuring functional connectivity using MEG: methodology and comparison with fcMRI. Neuroimage 56, 1082-1104.

Brookes, M.J., Liddle, E.B., Hale, J.R., Woolrich, M.W., Luckhoo, H., Liddle, P.F., Morris, P.G., 2012a. Task induced modulation of neural oscillations in electrophysiological brain networks. Neuroimage 63, 1918-1930.

1130 Brookes, M.J., O'Neill, G.C., Hall, E.L., Woolrich, M.W., Baker, A., Palazzo Corner, S., Robson, S.E., Morris, P.G., Barnes, G.R., 2014. Measuring temporal, spectral and spatial changes in electrophysiological brain network connectivity. Neuroimage 91, $282-$ 299.

Brookes, M.J., Tewarie, P.K., Hunt, B.A., Robson, S.E., Gascoyne, L.E., Liddle, E.B., Liddle, P.F., Morris, P.G., 2016. A multilayer network approach to MEG connectivity analysis. Neuroimage 132, 425-438.

Brookes, M.J., Woolrich, M., Luckhoo, H., Price, D., Hale, J.R., Stephenson, M.C., Barnes, G.R., Smith, S.M., Morris, P.G., 2011 b. Investigating the electrophysiological basis of resting state networks using magnetoencephalography. Proc Natl Acad Sci U S A $108,16783-16788$.

Brookes, M.J., Woolrich, M.W., Barnes, G.R., 2012b. Measuring functional connectivity in MEG: a multivariate approach insensitive to linear source leakage. Neuroimage 63, 910-920.

Brovelli, A., Badier, J.M., Bonini, F., Bartolomei, F., Coulon, O., Auzias, G., 2017. Dynamic Reconfiguration of Visuomotor-Related Functional Connectivity Networks. J Neurosci 37, 839-853.

Buice, M.A., Cowan, J.D., Chow, C.C., 2010. Systematic Fluctuation Expansion for Neural Network Activity Equations. Neural Computation 22, 377-426.

1150 Byrne, Á., Brookes, M.J., Coombes, S., 2017. A mean field model for movement induced changes in the beta rhythm. Journal of Computational Neuroscience In Press.

Cabral, J., Luckhoo, H., Woolrich, M., Joensson, M., Mohseni, H., Baker, A., Kringelbach, M.L., Deco, G., 2014. Exploring mechanisms of spontaneous functional connectivity in MEG: how delayed network interactions lead to structured amplitude envelopes of band-pass filtered oscillations. Neuroimage 90, 423-435.

Canolty, R.T., Edwards, E., Dalal, S.S., Soltani, M., Nagarajan, S.S., Kirsch, H.E., Berger, M.S., Barbaro, N.M., Knight, R.T., 2006. High gamma power is phase-locked to theta oscillations in human neocortex. Science 313, 1626-1628.

1160 Carbo, E.W., Hillebrand, A., van Dellen, E., Tewarie, P., de Witt Hamer, P.C., Baayen, J.C., Klein, M., Geurts, J.J., Reijneveld, J.C., Stam, C.J., Douw, L., 2017. Dynamic hub load predicts cognitive decline after resective neurosurgery. Sci Rep 7, 42117.

Cetin, M.S., Houck, J.M., Rashid, B., Agacoglu, O., Stephen, J.M., Sui, J., Canive, J., Mayer, A., Aine, C., Bustillo, J.R., Calhoun, V.D., 2016. Multimodal Classification of Schizophrenia Patients with MEG and fMRI Data Using Static and Dynamic Connectivity Measures. Front Neurosci 10, 466.

Chang, C., Glover, G.H., 2010. Time-frequency dynamics of resting-state brain connectivity measured with fMRI. Neuroimage $50,81-98$.

1170 Chang, C., Liu, Z., Chen, M.C., Liu, X., Duyn, J.H., 2013. EEG correlates of time-varying BOLD functional connectivity. Neuroimage 72, 227-236.

Cocchi, L., Zalesky, A., Toepel, U., Whitford, T.J., De-Lucia, M., Murray, M.M., Carter, O., 2011. Dynamic changes in brain functional connectivity during concurrent dual-task performance. PLoS One 6, e28301.

Cohen, D., 1972. Magnetoencephalography: detection of the brain's electrical activity with a superconducting magnetometer. Science 175, 664-666.

Coito, A., Plomp, G., Genetti, M., Abela, E., Wiest, R., Seeck, M., Michel, C.M., Vulliemoz, S., 2015. Dynamic directed interictal connectivity in left and right temporal lobe epilepsy. Epilepsia 56, 207-217.

Colclough, G.L., Brookes, M.J., Smith, S.M., Woolrich, M.W., 2015. A symmetric multivariate leakage correction for MEG connectomes. Neuroimage 117, 439-448.

Colclough, G.L., Woolrich, M.W., Tewarie, P.K., Brookes, M.J., Quinn, A.J., Smith, S.M., 2016. How reliable are MEG restingstate connectivity metrics? Neuroimage 138, 284-293.

Cole, M.W., Bassett, D.S., Power, J.D., Braver, T.S., Petersen, S.E., 2014. Intrinsic and task-evoked network architectures of the human brain. Neuron 83, 238-251.

Coombes, S., 2005. Waves, bumps, and patterns in neural field theories. Biological Cybernetics 93, 91-108. 
Coombes, S., beim Graben, P., Potthast, R., 2014a. Tutorial on Neural Field Theory. In: Coombes, S., beim Graben, P., Potthast, R., Wright, J. (Eds.), Neural Fields: Theory and Applications. Springer Berlin Heidelberg, Berlin, Heidelberg, pp. 1-43.

Coombes, S., beim Graben, P., Potthast, R., Wright, J., 2014b. Neural Fields: Theory and Applications. Springer.

Coombes, S., Byrne, Á., 2016. Next generation neural mass models. arXiv preprint arXiv:1607.06251.

1200 Cozzo, E., de Arruda, G.F., Rodrigues, F.A., Moreno, Y., 2016. Multilayer Networks: Metrics and Spectral Properties. In: Garas, A. (Ed.), Interconnected Networks. Springer International Publishing, Cham, pp. 17-35.

Crossley, N.A., Mechelli, A., Vertes, P.E., Winton-Brown, T.T., Patel, A.X., Ginestet, C.E., McGuire, P., Bullmore, E.T., 2013. Cognitive relevance of the community structure of the human brain functional coactivation network. Proc Natl Acad Sci U S A $110,11583-11588$.

Daffertshofer, A., van Wijk, B.C., 2011. On the Influence of Amplitude on the Connectivity between Phases. Front Neuroinform 5,6 .

1210 de Pasquale, F., Della Penna, S., Snyder, A.Z., Lewis, C., Mantini, D., Marzetti, L., Belardinelli, P., Ciancetta, L., Pizzella, V., Romani, G.L., Corbetta, M., 2010. Temporal dynamics of spontaneous MEG activity in brain networks. Proc Natl Acad Sci U S A $107,6040-6045$.

de Pasquale, F., Della Penna, S., Snyder, A.Z., Marzetti, L., Pizzella, V., Romani, G.L., Corbetta, M., 2012. A cortical core for dynamic integration of functional networks in the resting human brain. Neuron 74, 753-764.

de Pasquale, F., Della Penna, S., Sporns, O., Romani, G.L., Corbetta, M., 2016. A Dynamic Core Network and Global Efficiency in the Resting Human Brain. Cereb Cortex 26, 4015-4033.

1220 Deco, G., Cabral, J., Woolrich, M.W., Stevner, A.B.A., van Hartevelt, T.J., Kringelbach, M.L., 2017. Single or multiple frequency generators in on-going brain activity: A mechanistic whole-brain model of empirical MEG data. Neuroimage 152, 538-550.

Deco, G., Jirsa, V., McIntosh, A.R., Sporns, O., Kotter, R., 2009. Key role of coupling, delay, and noise in resting brain fluctuations (vol 106, pg 10302, 2009). Proceedings of the National Academy of Sciences of the United States of America 106, 12207-12208.

Deco, G., Jirsa, V.K., 2012. Ongoing cortical activity at rest: criticality, multistability, and ghost attractors. J Neurosci 32, 33663375.

Deco, G., Jirsa, V.K., McIntosh, A.R., 2011. Emerging concepts for the dynamical organization of resting-state activity in the brain. Nat Rev Neurosci 12, 43-56.

Deco, G., Jirsa, V.K., Robinson, P.A., Breakspear, M., Friston, K., 2008. The dynamic brain: from spiking neurons to neural masses and cortical fields. PLoS Comput Biol 4, e1000092.

Dhamala, M., Rangarajan, G., Ding, M., 2008. Analyzing information flow in brain networks with nonparametric Granger causality. Neuroimage 41, 354-362.

Dimitriadis, S.I., Laskaris, N.A., Simos, P.G., Micheloyannis, S., Fletcher, J.M., Rezaie, R., Papanicolaou, A.C., 2013. Altered temporal correlations in resting-state connectivity fluctuations in children with reading difficulties detected via MEG. Neuroimage 83, 307-317.

1240

Doron, K.W., Bassett, D.S., Gazzaniga, M.S., 2012. Dynamic network structure of interhemispheric coordination. Proc Natl Acad Sci U S A 109, 18661-18668.

Elton, A., Gao, W., 2015. Task-related modulation of functional connectivity variability and its behavioral correlations. Hum Brain Mapp 36, 3260-3272.

Engel, A.K., Gerloff, C., Hilgetag, C.C., Nolte, G., 2013. Intrinsic coupling modes: multiscale interactions in ongoing brain activity. Neuron 80, 867-886.

1250 Escudero, J., Acar, E., Fernandez, A., Bro, R., 2015. Multiscale entropy analysis of resting-state magnetoencephalogram with tensor factorisations in Alzheimer's disease. Brain Res Bull 119, 136-144.

Farahibozorg, S.-R., Henson, R.N., Hauk, O., 2017. Adaptive Cortical Parcellations for Source Reconstructed EEG/MEG Connectomes. bioRxiv.

Florin, E., Baillet, S., 2015. The brain's resting-state activity is shaped by synchronized cross-frequency coupling of neural oscillations. Neuroimage 111, 26-35.

Fox, M.D., Raichle, M.E., 2007. Spontaneous fluctuations in brain activity observed with functional magnetic resonance imaging. Nat Rev Neurosci 8, 700-711.

Fox, M.D., Snyder, A.Z., Vincent, J.L., Corbetta, M., Van Essen, D.C., Raichle, M.E., 2005. The human brain is intrinsically organized into dynamic, anticorrelated functional networks. Proceedings of the National Academy of Sciences of the United States of America 102, 9673-9678. 
Freestone, D.R., Aram, P., Dewar, M., Scerri, K., Grayden, D.B., Kadirkamanathan, V., 2011. A data-driven framework for neural field modeling. Neuroimage 56, 1043-1058.

Fries, P., 2015. Rhythms for Cognition: Communication through Coherence. Neuron 88, 220-235.

Friston, K.J., 1994. Functional and effective connectivity in neuroimaging: A synthesis. Human Brain Mapping 2, 56-78.

Friston, K.J., 1998. The disconnection hypothesis. Schizophr Res 30, 115-125.

Friston, K.J., 2011. Functional and effective connectivity: a review. Brain Connect 1, 13-36.

Gao, L., Sommerlade, L., Coffman, B., Zhang, T., Stephen, J.M., Li, D., Wang, J., Grebogi, C., Schelter, B., 2015. Granger causal time-dependent source connectivity in the somatosensory network. Sci Rep 5, 10399.

1280 Garcés, P., Martín-Buro, M.C., Maestú, F., 2016. Quantifying the Test-Retest Reliability of Magnetoencephalography RestingState Functional Connectivity. Brain Connectivity 6, 448-460.

Gauvin, L., Panisson, A., Cattuto, C., 2014. Detecting the community structure and activity patterns of temporal networks: a nonnegative tensor factorization approach. PLoS One 9, e86028.

Goñi, J., van den Heuvel, M.P., Avena-Koenigsberger, A., Velez de Mendizabal, N., Betzel, R.F., Griffa, A., Hagmann, P., Corominas-Murtra, B., Thiran, J.P., Sporns, O., 2014. Resting-brain functional connectivity predicted by analytic measures of network communication. Proc Natl Acad Sci U S A 111, 833-838.

1290 Grandjean, J., Preti, M.G., Bolton, T.A., Buerge, M., Seifritz, E., Pryce, C.R., Van De Ville, D., Rudin, M., 2017. Dynamic reorganization of intrinsic functional networks in the mouse brain. Neuroimage 152, 497-508.

Granger, C.W.J., 1969. Investigating Causal Relations by Econometric Models and Cross-Spectral Methods. Econometrica 37, 414-\&.

Grinsted, A., Moore, J.C., Jevrejeva, S., 2004. Application of the cross wavelet transform and wavelet coherence to geophysical time series. Nonlinear Processes in Geophysics 11, 561-566.

1300

Haimovici, A., Tagliazucchi, E., Balenzuela, P., Chialvo, D.R., 2013. Brain Organization into Resting State Networks Emerges at Criticality on a Model of the Human Connectome. Physical Review Letters 110

Hall, E.L., Woolrich, M.W., Thomaz, C.E., Morris, P.G., Brookes, M.J., 2013. Using variance information in magnetoencephalography measures of functional connectivity. Neuroimage 67, 203-212.

Hampson, M., Driesen, N.R., Skudlarski, P., Gore, J.C., Constable, R.T., 2006. Brain connectivity related to working memory performance. J Neurosci 26, 13338-13343.

Hansen, E.C., Battaglia, D., Spiegler, A., Deco, G., Jirsa, V.K., 2015. Functional connectivity dynamics: modeling the switching behavior of the resting state. Neuroimage 105, 525-535.

1310

Hassan, M., Benquet, P., Biraben, A., Berrou, C., Dufor, O., Wendling, F., 2015. Dynamic reorganization of functional brain networks during picture naming. Cortex 73, 276-288.

Hassan, M., Merlet, I., Mheich, A., Kabbara, A., Biraben, A., Nica, A., Wendling, F., 2017. Identification of Interictal Epileptic Networks from Dense-EEG. Brain Topogr 30, 60-76.

Hauk, O., Stenroos, M., 2014. A framework for the design of flexible cross-talk functions for spatial filtering of EEG/MEG data: DeFleCT. Hum Brain Mapp 35, 1642-1653.

1320 Hauk, O., Wakeman, D.G., Henson, R., 2011. Comparison of noise-normalized minimum norm estimates for MEG analysis using multiple resolution metrics. Neuroimage 54, 1966-1974.

He, B.Y.J., 2014. Scale-free brain activity: past, present, and future. Trends in Cognitive Sciences 18, 480-487.

He, B.Y.J., Zempel, J.M., Snyder, A.Z., Raichle, M.E., 2010. The Temporal Structures and Functional Significance of Scale-free Brain Activity. Neuron 66, 353-369.

Hindriks, R., Adhikari, M.H., Murayama, Y., Ganzetti, M., Mantini, D., Logothetis, N.K., Deco, G., 2016. Can sliding-window correlations reveal dynamic functional connectivity in resting-state fMRI? Neuroimage 127, 242-256.

Hindriks, R., van Putten, M.J., 2013. Thalamo-cortical mechanisms underlying changes in amplitude and frequency of human alpha oscillations. Neuroimage 70, 150-163.

Hipp, J.F., Hawellek, D.J., Corbetta, M., Siegel, M., Engel, A.K., 2012. Large-scale cortical correlation structure of spontaneous oscillatory activity. Nat Neurosci 15, 884-890.

Hlinka, J., Coombes, S., 2012. Using computational models to relate structural and functional brain connectivity. European Journal of Neuroscience 36, 2137-2145. 
Honey, C.J., Kotter, R., Breakspear, M., Sporns, O., 2007. Network structure of cerebral cortex shapes functional connectivity on multiple time scales. Proceedings of the National Academy of Sciences of the United States of America 104, 10240-10245.

Huang, M.X., Shih, J.J., Lee, R.R., Harrington, D.L., Thoma, R.J., Weisend, M.P., Hanlon, F., Paulson, K.M., Li, T., Martin, K., Miller, G.A., Canive, J.M., 2004. Commonalities and differences among vectorized beamformers in electromagnetic source imaging. Brain Topography 16, 139-158.

Hunt, B.A., Tewarie, P.K., Mougin, O.E., Geades, N., Jones, D.K., Singh, K.D., Morris, P.G., Gowland, P.A., Brookes, M.J., 2016. Relationships between cortical myeloarchitecture and electrophysiological networks. Proc Natl Acad Sci U S A 113, 13510-13515.

Hunt, L.T., Kolling, N., Soltani, A., Woolrich, M.W., Rushworth, M.F., Behrens, T.E., 2012. Mechanisms underlying cortical activity during value-guided choice. Nat Neurosci 15, 470-476, S471-473.

Hutchison, R.M., Womelsdorf, T., Allen, E.A., Bandettini, P.A., Calhoun, V.D., Corbetta, M., Della Penna, S., Duyn, J.H., Glover, G.H., Gonzalez-Castillo, J., Handwerker, D.A., Keilholz, S., Kiviniemi, V., Leopold, D.A., de Pasquale, F., Sporns, O., Walter, M., Chang, C., 2013. Dynamic functional connectivity: promise, issues, and interpretations. Neuroimage 80, 360-378.

Hutt, A., Daffertshofer, A., Steinmetz, U., 2003. Detection of mutual phase synchronization in multivariate signals and application to phase ensembles and chaotic data. Physical Review E 68.

Izhikevich, E.M., Edelman, G.M., 2008. Large-scale model of mammalian thalamocortical systems. Proc Natl Acad Sci U S A 105, 3593-3598.

Johnson, S., Prendergast, G., Hymers, M., Green, G., 2011. Examining the effects of one- and three-dimensional spatial filtering analyses in magnetoencephalography. PLoS One 6, e22251.

Jones, S.R., Pinto, D.J., Kaper, T.J., Kopell, N., 2000. Alpha-frequency rhythms desynchronize over long cortical distances: a modeling study. J Comput Neurosci 9, 271-291.

1370

Kabbara, A., El Falou, W., Khalil, M., Wendling, F., Hassan, M., 2017. The dynamic functional core network of the human brain at rest. Scientific Reports 7.

Kessler, D., Angstadt, M., Welsh, R.C., Sripada, C., 2014. Modality-spanning deficits in attention-deficit/hyperactivity disorder in functional networks, gray matter, and white matter. J Neurosci 34, 16555-16566.

Khambhati, A.N., Bassett, D.S., Oommen, B.S., Chen, S.H., Lucas, T.H., Davis, K.A., Litt, B., 2017. Recurring Functional Interactions Predict Network Architecture of Interictal and Ictal States in Neocortical Epilepsy. eNeuro 4.

1380 Kivelä, M., Arenas, A., Barthelemy, M., Gleeson, J.P., Moreno, Y., Porter, M.A., 2014. Multilayer networks. Journal of Complex Networks 2, 203-271.

Knyazev, G.G., Savostyanov, A.N., Bocharov, A.V., Tamozhnikov, S.S., Saprigyn, A.E., 2016. Task-positive and task-negative networks and their relation to depression: EEG beamformer analysis. Behav Brain Res 306, 160-169.

Koelewijn, L., Bompas, A., Tales, A., Brookes, M.J., Muthukumaraswamy, S.D., Bayer, A., Singh, K.D., 2017. Alzheimer's disease disrupts alpha and beta-band resting-state oscillatory network connectivity. Clin Neurophysiol.

Koelewijn, L., Hamandi, K., Brindley, L.M., Brookes, M.J., Routley, B.C., Muthukumaraswamy, S.D., Williams, N., Thomas, M.A., Kirby, A., Te Water Naude, J., Gibbon, F., Singh, K.D., 2015. Resting-state oscillatory dynamics in sensorimotor cortex in benign epilepsy with centro-temporal spikes and typical brain development. Hum Brain Mapp 36, 3935-3949.

Kucyi, A., Davis, K.D., 2014. Dynamic functional connectivity of the default mode network tracks daydreaming. Neuroimage 100 , 471-480.

Lachaux, J.P., Rodriguez, E., Martinerie, J., Varela, F.J., 1999. Measuring phase synchrony in brain signals. Hum Brain Mapp 8 , 194-208.

Lackner, C.L., Marshall, W.J., Santesso, D.L., Dywan, J., Wade, T., Segalowitz, S.J., 2014. Adolescent anxiety and aggression can be differentially predicted by electrocortical phase reset variables. Brain Cogn 89, 90-98.

Lamme, V.A., 2003. Why visual attention and awareness are different. Trends Cogn Sci 7, 12-18.

Le Van Quyen, M., Martinerie, J., Baulac, M., Varela, F., 1999. Anticipating epileptic seizures in real time by a non-linear analysis of similarity between EEG recordings. Neuroreport 10, 2149-2155.

Lee, H., Noh, G.-J., Joo, P., Choi, B.-M., Silverstein, B.H., Kim, M., Wang, J., Jung, W.-S., Kim, S., 2017. Diversity of functional connectivity patterns is reduced in propofol-induced unconsciousness. Human Brain Mapping.

1410 Leonardi, N., Richiardi, J., Gschwind, M., Simioni, S., Annoni, J.M., Schluep, M., Vuilleumier, P., Van De Ville, D., 2013. Principal components of functional connectivity: a new approach to study dynamic brain connectivity during rest. Neuroimage 83, 937-950.

Liddle, E.B., Price, D., Palaniyappan, L., Brookes, M.J., Robson, S.E., Hall, E.L., Morris, P.G., Liddle, P.F., 2016. Abnormal salience signaling in schizophrenia: The role of integrative beta oscillations. Human Brain Mapping 37, 1361-1374. 
Liégeois, R., Laumann, T.O., Snyder, A.Z., Zhou, H.J., Yeo, B.T.T., 2017. Interpreting Temporal Fluctuations In Resting-State Functional Connectivity MRI. bioRxiv.

Linden, H., Pettersen, K.H., Einevoll, G.T., 2010. Intrinsic dendritic filtering gives low-pass power spectra of local field potentials. Journal of Computational Neuroscience 29, 423-444.

Lindquist, M.A., Xu, Y., Nebel, M.B., Caffo, B.S., 2014. Evaluating dynamic bivariate correlations in resting-state fMRI: a comparison study and a new approach. Neuroimage 101, 531-546.

Linkenkaer-Hansen, K., Nikouline, V.V., Palva, J.M., Ilmoniemi, R.J., 2001. Long-range temporal correlations and scaling behavior in human brain oscillations. J Neurosci 21, 1370-1377.

Liu, Z., Fukunaga, M., de Zwart, J.A., Duyn, J.H., 2010. Large-scale spontaneous fluctuations and correlations in brain electrical activity observed with magnetoencephalography. Neuroimage 51, 102-111.

Liuzzi, L., Gascoyne, L.E., Tewarie, P.K., Barratt, E.L., Boto, E., Brookes, M.J., 2016. Optimising experimental design for MEG resting state functional connectivity measurement. Neuroimage.

Lowdin, P.O., 1950. On the Non-Orthogonality Problem Connected with the Use of Atomic Wave Functions in the Theory of Molecules and Crystals. Journal of Chemical Physics 18, 365-375.

Lu, Q., Bi, K., Liu, C., Luo, G., Tang, H., Yao, Z., 2013. Predicting depression based on dynamic regional connectivity: a windowed Granger causality analysis of MEG recordings. Brain Res 1535, 52-60.

1440 Luckhoo, H., Hale, J.R., Stokes, M.G., Nobre, A.C., Morris, P.G., Brookes, M.J., Woolrich, M.W., 2012. Inferring task-related networks using independent component analysis in magnetoencephalography. Neuroimage 62, 530-541.

MacDonald, K.D., Brett, B., Barth, D.S., 1996. Inter- and intra-hemispheric spatiotemporal organization of spontaneous electrocortical oscillations. J Neurophysiol 76, 423-437.

MacQueen, J., 1967. Some methods for classification and analysis of multivariate observations. Proceedings of the Fifth Berkeley Symposium on Mathematical Statistics and Probability, Volume 1: Statistics. University of California Press, Berkeley, Calif., pp. 281-297.

1450 Maldjian, J.A., Davenport, E.M., Whitlow, C.T., 2014. Graph theoretical analysis of resting-state MEG data: Identifying interhemispheric connectivity and the default mode. Neuroimage 96, 88-94.

Maris, E., Oostenveld, R., 2007. Nonparametric statistical testing of EEG- and MEG-data. J Neurosci Methods 164, 177-190.

Martini, N., Menicucci, D., Sebastiani, L., Bedini, R., Pingitore, A., Vanello, N., Milanesi, M., Landini, L., Gemignani, A., 2012. The dynamics of EEG gamma responses to unpleasant visual stimuli: from local activity to functional connectivity. Neuroimage 60, 922-932.

Marzetti, L., Della Penna, S., Snyder, A.Z., Pizzella, V., Nolte, G., de Pasquale, F., Romani, G.L., Corbetta, M., 2013. Frequency specific interactions of MEG resting state activity within and across brain networks as revealed by the multivariate interaction measure. Neuroimage 79, 172-183.

Meier, J., Tewarie, P., Hillebrand, A., Douw, L., van Dijk, B.W., Stufflebeam, S.M., Van Mieghem, P., 2016. A Mapping Between Structural and Functional Brain Networks. Brain Connect 6, 298-311.

Meier, J., Zhou, X., Hillebrand, A., Tewarie, P., Stam, C.J., Van Mieghem, P., 2017. The epidemic spreading model and the direction of information flow in brain networks. Neuroimage 152, 639-646.

Messe, A., Rudrauf, D., Giron, A., Marrelec, G., 2015. Predicting functional connectivity from structural connectivity via computational models using MRI: An extensive comparison study. Neuroimage 111, 65-75.

Meunier, D., Lambiotte, R., Fornito, A., Ersche, K.D., Bullmore, E.T., 2009. Hierarchical modularity in human brain functional networks. Front Neuroinform 3, 37.

Miller, K.J., Sorensen, L.B., Ojemann, J.G., den Nijs, M., 2009. Power-Law Scaling in the Brain Surface Electric Potential. Plos Computational Biology 5.

Misic, B., Betzel, R.F., Nematzadeh, A., Goni, J., Griffa, A., Hagmann, P., Flammini, A., Ahn, Y.Y., Sporns, O., 2015. Cooperative and Competitive Spreading Dynamics on the Human Connectome. Neuron 86, 1518-1529.

Moon, J.Y., Lee, U., Blain-Moraes, S., Mashour, G.A., 2015. General relationship of global topology, local dynamics, and directionality in large-scale brain networks. PLoS Comput Biol 11, e1004225.

Mucha, P.J., Richardson, T., Macon, K., Porter, M.A., Onnela, J.P., 2010. Community Structure in Time-Dependent, Multiscale, and Multiplex Networks. Science 328, 876-878.

Nakagawa, T.T., Woolrich, M., Luckhoo, H., Joensson, M., Mohseni, H., Kringelbach, M.L., Jirsa, V., Deco, G., 2014. How delays matter in an oscillatory whole-brain spiking-neuron network model for MEG alpha-rhythms at rest. Neuroimage 87, 383-394. 
Nicosia, V., Tang, J., Mascolo, C., Musolesi, M., Russo, G., Latora, V., 2013. Graph Metrics for Temporal Networks. In: Holme, P., Saramäki, J. (Eds.), Temporal Networks. Springer Berlin Heidelberg, Berlin, Heidelberg, pp. 15-40.

Nolte, G., Bai, O., Wheaton, L., Mari, Z., Vorbach, S., Hallett, M., 2004. Identifying true brain interaction from EEG data using the imaginary part of coherency. Clin Neurophysiol 115, 2292-2307.

Nolte, G., Ziehe, A., Nikulin, V.V., Schlogl, A., Kramer, N., Brismar, T., Muller, K.R., 2008. Robustly estimating the flow direction of information in complex physical systems. Phys Rev Lett 100, 234101.

Nugent, A.C., Robinson, S.E., Coppola, R., Furey, M.L., Zarate, C.A., Jr., 2015. Group differences in MEG-ICA derived resting state networks: Application to major depressive disorder. Neuroimage 118, 1-12.

Nunez, P.L., Srinivasan, R., Westdorp, A.F., Wijesinghe, R.S., Tucker, D.M., Silberstein, R.B., Cadusch, P.J., 1997. EEG coherency: I: statistics, reference electrode, volume conduction, Laplacians, cortical imaging, and interpretation at multiple scales. Electroencephalography and Clinical Neurophysiology 103, 499-515.

1510

'Dea, R., Crofts, J.J., Kaiser, M., 2013. Spreading dynamics on spatially constrained complex brain networks. Journal of the Royal Society Interface 10

O'Neill, G.C., Barratt, E.L., Hunt, B.A., Tewarie, P.K., Brookes, M.J., 2015a. Measuring electrophysiological connectivity by power envelope correlation: a technical review on MEG methods. Phys Med Biol 60, R271-295.

O'Neill, G.C., Bauer, M., Woolrich, M.W., Morris, P.G., Barnes, G.R., Brookes, M.J., 2015b. Dynamic recruitment of resting state sub-networks. Neuroimage 115, 85-95.

O'Neill, G.C., Tewarie, P.K., Colclough, G.L., Gascoyne, L.E., Hunt, B.A., Morris, P.G., Woolrich, M.W., Brookes, M.J., 2017. Measurement of dynamic task related functional networks using MEG. Neuroimage 146, 667-678.

1520 Palaniyappan, L., Liddle, P.F., 2012. Does the salience network play a cardinal role in psychosis? An emerging hypothesis of insular dysfunction. J Psychiatry Neurosci 37, 17-27.

Paluš, M., 1997. Detecting phase synchronization in noisy systems. Physics Letters A 235, 341-351.

Palva, S., Palva, J.M., 2012. Discovering oscillatory interaction networks with M/EEG: challenges and breakthroughs. Trends Cogn Sci 16, 219-230.

Peled, A., Geva, A.B., Kremen, W.S., Blankfeld, H.M., Esfandiarfard, R., Nordahl, T.E., 2001. Functional connectivity and working memory in schizophrenia: an EEG study. Int J Neurosci 106, 47-61.

1530

Penfield, W., Jasper, H., 1954. Epilepsy and the functional anatomy of the human brain.

Pereda, E., Quiroga, R.Q., Bhattacharya, J., 2005. Nonlinear multivariate analysis of neurophysiological signals. Prog Neurobiol 77, 1-37.

Petkoski, S., Spiegler, A., Proix, T., Aram, P., Temprado, J.J., Jirsa, V.K., 2016. Heterogeneity of time delays determines synchronization of coupled oscillators. Physical Review E 94.

1540

Pfurtscheller, G., Aranibar, A., 1977. Event-Related Cortical Desynchronization Detected by Power Measurements of Scalp Eeg. Electroencephalography and Clinical Neurophysiology 42, 817-826.

Pfurtscheller, G., Lopes da Silva, F.H., 1999. Event-related EEG/MEG synchronization and desynchronization: basic principles. Clin Neurophysiol 110, 1842-1857.

Poil, S.S., Hardstone, R., Mansvelder, H.D., Linkenkaer-Hansen, K., 2012. Critical-State Dynamics of Avalanches and Oscillations Jointly Emerge from Balanced Excitation/Inhibition in Neuronal Networks. Journal of Neuroscience 32, 9817-9823.

Poil, S.S., van Ooyen, A., Linkenkaer-Hansen, K., 2008. Avalanche dynamics of human brain oscillations: relation to critical branching processes and temporal correlations. Hum Brain Mapp 29, 770-777.

Ponce-Alvarez, A., Deco, G., Hagmann, P., Romani, G.L., Mantini, D., Corbetta, M., 2015. Resting-state temporal synchronization networks emerge from connectivity topology and heterogeneity. PLoS Comput Biol 11, e1004100.

Preti, M.G., Bolton, T.A., Van De Ville, D., 2016. The dynamic functional connectome: State-of-the-art and perspectives. Neuroimage.

Prichard, D., Theiler, J., 1994. Generating Surrogate Data for Time-Series with Several Simultaneously Measured Variables. Physical Review Letters 73, 951-954.

1560 Rabiner, L.R., 1989. A Tutorial on Hidden Markov-Models and Selected Applications in Speech Recognition. Proceedings of the leee $77,257-286$.

Raichle, M.E., MacLeod, A.M., Snyder, A.Z., Powers, W.J., Gusnard, D.A., Shulman, G.L., 2001. A default mode of brain function. Proc Natl Acad Sci U S A 98, 676-682. 
Robinson, P.A., 2012. Interrelating anatomical, effective, and functional brain connectivity using propagators and neural field theory. Physical Review E 85.

Robinson, P.A., Rennie, C.J., Rowe, D.L., 2002. Dynamics of large-scale brain activity in normal arousal states and epileptic seizures. Phys Rev E Stat Nonlin Soft Matter Phys 65, 041924.

Robinson, P.A., Rennie, C.J., Wright, J.J., Bahramali, H., Gordon, E., Rowe, D.L., 2001. Prediction of electroencephalographic spectra from neurophysiology. Physical Review E 63.

Robinson, P.A., Sarkar, S., Pandejee, G.M., Henderson, J.A., 2014. Determination of effective brain connectivity from functional connectivity with application to resting state connectivities. Physical Review E 90.

Saggio, M.L., Ritter, P., Jirsa, V.K., 2016. Analytical Operations Relate Structural and Functional Connectivity in the Brain. PLoS One 11.

1580

Samiee, S., Baillet, S., 2017. Time-resolved phase-amplitude coupling in neural oscillations. Neuroimage.

Schnitzler, A., Gross, J., 2005. Normal and pathological oscillatory communication in the brain. Nat Rev Neurosci 6, 285-296.

Schoffelen, J.M., Gross, J., 2009. Source connectivity analysis with MEG and EEG. Hum Brain Mapp 30, 1857-1865.

Schoonheim, M.M., Meijer, K.A., Geurts, J.J., 2015. Network collapse and cognitive impairment in multiple sclerosis. Front Neurol 6, 82 .

1590 Schreiber, T., 2000. Measuring information transfer. Physical Review Letters 85, 461-464.

Senden, M., Deco, G., de Reus, M.A., Goebel, R., van den Heuvel, M.P., 2014. Rich club organization supports a diverse set of functional network configurations. Neuroimage 96, 174-182.

Shulman, G.L., Fiez, J.A., Corbetta, M., Buckner, R.L., Miezin, F.M., Raichle, M.E., Petersen, S.E., 1997. Common Blood Flow Changes across Visual Tasks: II. Decreases in Cerebral Cortex. J Cogn Neurosci 9, 648-663.

Singer, W., 1999. Neuronal synchrony: a versatile code for the definition of relations? Neuron 24, 49-65, 111-125. Sizemore, A.E., Bassett, D.S., 2017. Dynamic Graph Metrics: Tutorial, Toolbox, and Tale. arXiv preprint arXiv:1703.10643.

1600

Smith, S.M., Fox, P.T., Miller, K.L., Glahn, D.C., Fox, P.M., Mackay, C.E., Filippini, N., Watkins, K.E., Toro, R., Laird, A.R., Beckmann, C.F., 2009. Correspondence of the brain's functional architecture during activation and rest. Proc Natl Acad Sci U S A $106,13040-13045$

Stam, C.J., 2004. Functional connectivity patterns of human magnetoencephalographic recordings: a 'small-world' network? Neurosci Lett 355, 25-28.

Stam, C.J., Breakspear, M., van Cappellen van Walsum, A.M., van Dijk, B.W., 2003. Nonlinear synchronization in EEG and whole-head MEG recordings of healthy subjects. Hum Brain Mapp 19, 63-78.

1610

Stam, C.J., Montez, T., Jones, B.F., Rombouts, S.A., van der Made, Y., Pijnenburg, Y.A., Scheltens, P., 2005. Disturbed fluctuations of resting state EEG synchronization in Alzheimer's disease. Clin Neurophysiol 116, 708-715.

Stam, C.J., Nolte, G., Daffertshofer, A., 2007. Phase lag index: assessment of functional connectivity from multi channel EEG and MEG with diminished bias from common sources. Hum Brain Mapp 28, 1178-1193.

Stam, C.J., van Dijk, B.W., 2002. Synchronization likelihood: an unbiased measure of generalized synchronization in multivariate data sets. Physica D: Nonlinear Phenomena 163, 236-251.

1620 Stam, C.J., van Straaten, E.C., Van Dellen, E., Tewarie, P., Gong, G., Hillebrand, A., Meier, J., Van Mieghem, P., 2016. The relation between structural and functional connectivity patterns in complex brain networks. Int J Psychophysiol 103, 149-160.

Stefanescu, R.A., Jirsa, V.K., 2008. A Low Dimensional Description of Globally Coupled Heterogeneous Neural Networks of Excitatory and Inhibitory Neurons. Plos Computational Biology 4.

Stevens, M.C., 2016. The contributions of resting state and task-based functional connectivity studies to our understanding of adolescent brain network maturation. Neuroscience and Biobehavioral Reviews 70, 13-32.

Tagliazucchi, E., Siniatchkin, M., Laufs, H., Chialvo, D.R., 2016. The Voxel-Wise Functional Connectome Can Be Efficiently Derived from Co-activations in a Sparse Spatio-Temporal Point-Process. Front Neurosci 10, 381

Tagliazucchi, E., von Wegner, F., Morzelewski, A., Brodbeck, V., Laufs, H., 2012. Dynamic BOLD functional connectivity in humans and its electrophysiological correlates. Front Hum Neurosci 6, 339.

Taulu, S., Kajola, M., Simola, J., 2004. Suppression of interference and artifacts by the signal space separation method. Brain Topography 16, 269-275.

Tewarie, P., Hillebrand, A., van Dellen, E., Schoonheim, M.M., Barkhof, F., Polman, C.H., Beaulieu, C., Gong, G., van Dijk, B.W., Stam, C.J., 2014. Structural degree predicts functional network connectivity: a multimodal resting-state fMRI and MEG study. Neuroimage 97, 296-307. 
Tewarie, P., Hillebrand, A., van Dijk, B.W., Stam, C.J., O'Neill, G.C., Van Mieghem, P., Meier, J.M., Woolrich, M.W., Morris, P.G., Brookes, M.J., 2016. Integrating cross-frequency and within band functional networks in resting-state MEG: A multi-layer network approach. Neuroimage 142, 324-336.

Tewarie, P., van Dellen, E., Hillebrand, A., Stam, C.J., 2015. The minimum spanning tree: an unbiased method for brain network analysis. Neuroimage 104, 177-188.

Thatcher, R.W., North, D.M., Biver, C.J., 2009. Self-organized criticality and the development of EEG phase reset. Human Brain Mapping 30, 553-574.

Tijms, B.M., Wink, A.M., de Haan, W., van der Flier, W.M., Stam, C.J., Scheltens, P., Barkhof, F., 2013. Alzheimer's disease: connecting findings from graph theoretical studies of brain networks. Neurobiol Aging 34, 2023-2036.

Tinker, J., Velazquez, J.L., 2014. Power law scaling in synchronization of brain signals depends on cognitive load. Front Syst Neurosci 8, 73.

Ton, R., Deco, G., Daffertshofer, A., 2014. Structure-Function Discrepancy: Inhomogeneity and Delays in Synchronized Neural Networks. Plos Computational Biology 10.

1660

Ton, R., Deco, G., Kringelbach, M.L., Woolrich, M., Daffertshofer, A., 2015. Distinct criticality of phase and amplitude dynamics in the resting brain. arXiv preprint arXiv:1512.02574.

van den Heuvel, M.P., de Lange, S.C., Zalesky, A., Seguin, C., Yeo, B.T.T., Schmidt, R., 2017. Proportional thresholding in resting-state fMRI functional connectivity networks and consequences for patient-control connectome studies: Issues and recommendations. Neuroimage 152, 437-449.

van Diessen, E., Diederen, S.J., Braun, K.P., Jansen, F.E., Stam, C.J., 2013. Functional and structural brain networks in epilepsy: what have we learned? Epilepsia 54, 1855-1865

1670

Van Mieghem, P., 2009. Performance analysis of communications networks and systems. Cambridge University Press.

van Wijk, B.C.M., Stam, C.J., Daffertshofer, A., 2010. Comparing Brain Networks of Different Size and Connectivity Density Using Graph Theory. PLoS One 5.

Vidaurre, D., Abeysuriya, R., Becker, R., Quinn, A.J., Alfaro-Almagro, F., Smith, S.M., Woolrich, M.W., 2017a. Discovering dynamic brain networks from big data in rest and task. Neuroimage.

Vidaurre, D., Hunt, L.T., Quinn, A.J., Hunt, B.A.E., Brookes, M.J., Nobre, A.C., Woolrich, M.W., 2017b. Spontaneous cortical activity transiently organises into frequency specific phase-coupling networks. bioRxiv.

Vidaurre, D., Quinn, A.J., Baker, A.P., Dupret, D., Tejero-Cantero, A., Woolrich, M.W., 2016. Spectrally resolved fast transient brain states in electrophysiological data. Neuroimage 126, 81-95.

Vinck, M., Oostenveld, R., van Wingerden, M., Battaglia, F., Pennartz, C.M., 2011. An improved index of phase-synchronization for electrophysiological data in the presence of volume-conduction, noise and sample-size bias. Neuroimage 55, 1548-1565.

Wang, H.E., Benar, C.G., Quilichini, P.P., Friston, K.J., Jirsa, V.K., Bernard, C., 2014. A systematic framework for functional connectivity measures. Front Neurosci 8, 405.

1690

Wens, V., Marty, B., Mary, A., Bourguignon, M., Op de Beeck, M., Goldman, S., Van Bogaert, P., Peigneux, P., De Tiege, X., 2015. A geometric correction scheme for spatial leakage effects in $M E G / E E G$ seed-based functional connectivity mapping. Hum Brain Mapp 36, 4604-4621.

Wen, H., Liu, Z., 2016. Broadband Electrophysiological Dynamics Contribute to Global Resting-State fMRI Signal. J Neurosci 36, 6030-6040.

Wiesman, A.I., Heinrichs-Graham, E., Coolidge, N.M., Gehringer, J.E., Kurz, M.J., Wilson, T.W., 2017. Oscillatory dynamics and functional connectivity during gating of primary somatosensory responses. The Journal of Physiology 595, 1365-1375.

1700

Winkler, A.M., Ridgway, G.R., Webster, M.A., Smith, S.M., Nichols, T.E., 2014. Permutation inference for the general linear model. Neuroimage 92, 381-397.

Xu, Y., Lindquist, M.A., 2015. Dynamic connectivity detection: an algorithm for determining functional connectivity change points in fMRI data. Front Neurosci 9, 285.

Yang, C.Y., Chao, Y.P., Lin, C.P., 2012. Differences in Early Dynamic Connectivity between Visual Expansion and Contraction Stimulations Revealed by an fMRI-Directed MEG Approach. Journal of Neuroimaging 22, 400-407.

1710 Zalesky, A., Fornito, A., Cocchi, L., Gollo, L.L., Breakspear, M., 2014. Time-resolved resting-state brain networks. Proc Natl Acad Sci U S A 111, 10341-10346. 